\title{
Isoform Specificity of PKMs during Long-Term Facilitation in Aplysia Is Mediated through Stabilization by KIBRA
}

\author{
Larissa Ferguson, ${ }^{1}$ OJiangyuan $\mathrm{Hu},{ }^{2}$ Diancai Cai, ${ }^{3}$ Shanping Chen, ${ }^{3}$ Tyler W. Dunn, ${ }^{1}$ Kaycey Pearce, ${ }^{3}$ \\ David L. Glanzman, ${ }^{3,4,5,6}$ @Samuel Schacher, ${ }^{2}$ and ${ }^{-W}$ Wayne S. Sossin ${ }^{1}$ \\ ${ }^{1}$ Department of Neurology and Neurosurgery, Montreal Neurological Institute, McGill University, Montreal, Quebec H3A 2B4, Canada, ${ }^{2}$ Department of \\ Neuroscience, Columbia University College of Physicians and Surgeons, New York State Psychiatric Institute, New York, New York 10032, ${ }^{3}$ Department of \\ Integrative Biology and Physiology, College of Letters and Science, ${ }^{4}$ Department of Neurobiology, ${ }^{5}$ Brain Research Institute, and ${ }^{6}$ Integrative Center for \\ Learning and Memory, David Geffen School of Medicine, University of California at Los Angeles, Los Angeles, California 90095
}

Persistent activity of protein kinase $\mathrm{M}(\mathrm{PKM})$, the truncated form of protein kinase $\mathrm{C}(\mathrm{PKC})$, can maintain long-term changes in synaptic strength in many systems, including the hermaphrodite marine mollusk, Aplysia californica. Moreover, different types of long-term facilitation (LTF) in cultured Aplysia sensorimotor synapses rely on the activities of different PKM isoforms in the presynaptic sensory neuron and postsynaptic motor neuron. When the atypical PKM isoform is required, the kidney and brain expressed adaptor protein (KIBRA) is also required. Here, we explore how this isoform specificity is established. We find that PKM overexpression in the motor neuron, but not the sensory neuron, is sufficient to increase synaptic strength and that this activity is not isoform-specific. KIBRA is not the rate-limiting step in facilitation since overexpression of KIBRA is neither sufficient to increase synaptic strength, nor to prolong a form of PKM-dependent intermediate synaptic facilitation. However, the isoform specificity of dominant-negative-PKMs to erase LTF is correlated with isoform-specific competition for stabilization by KIBRA. We identify a new conserved region of KIBRA. Different splice isoforms in this region stabilize different PKMs based on the isoform-specific sequence of an $\alpha$-helix "handle" in the PKMs. Thus, specific stabilization of distinct PKMs by different isoforms of KIBRA can explain the isoform specificity of PKMs during LTF in Aplysia.

Key words: Aplysia; KIBRA; kinase; long-term facilitation; PKM; synaptic plasticity

Significance Statement

Long-lasting changes in synaptic plasticity associated with memory formation are maintained by persistent protein kinases. We have previously shown in the Aplysia sensorimotor model that distinct isoforms of persistently active protein kinase Cs (PKMs) maintain distinct forms of long-lasting synaptic changes, even when both forms are expressed in the same motor neuron. Here, we show that, while the effects of overexpression of PKMs are not isoform-specific, isoform specificity is defined by a "handle" helix in PKMs that confers stabilization by distinct splice forms in a previously undefined domain of the adaptor protein KIBRA. Thus, we define new regions in both KIBRA and PKMs that define the isoform specificity for maintaining synaptic strength in distinct facilitation paradigms.

\section{Introduction}

The long-term changes in synaptic strength that underlie memory can be maintained through persistently active kinases

\footnotetext{
Received April 26, 2019; revised July 29, 2019; accepted Aug. 3, 2019.

Author contributions: L.F., J.H., S.C., D.L.G., S.S., and W.S.S. designed research; L.F., J.H., D.C., S.C., T.W.D., and K.P. performed research; L.F., J.H., D.C., T.W.D., and W.S.S. analyzed data; L.F. and W.S.S. wrote the first draft of the paper; L.F., J.H., T.W.D., D.L.G., S.S., and W.S.S. edited the paper.

This work was supported by Canadian Institutes of Health Research Grant 340328 to W.S.S., F.S.B. Miller Fellowship to L.F., National Institutes of Health Grant MH-060387 to J.H. and S.S., National Institutes of Health Grants R01 NS02956 and R01 MH096120, and National Science Foundation Grant IOS 1121690 to D.L.G. W.S.S. is a James McGill Professor.

The authors declare no competing financial interests.

Correspondence should be addressed to Wayne S. Sossin at wayne.sossin@mcgill.ca.
}

(Greenberg et al., 1987; Lisman, 1994; Sacktor, 2011; Sossin, 2018). One such kinase is protein kinase M (PKM), the truncated form of protein kinase C (PKC). PKCs are classified into three families (conventional, novel, and atypical), which differ mainly through the structure of their regulatory domain (Sossin, 2007). While much attention has been given to the role of the atypical $\mathrm{PKM} \zeta$ in memory maintenance (Sacktor, 2011), the fact that the catalytic domains of PKC/PKM families are similar in structure suggests that additional PKM isoforms may perform similar functions. $\mathrm{PKM} \zeta \mathrm{KO}$ mice, for example, are phenotypically nor- 
mal due to compensation by the closely related $\iota$ isoform (Tsokas et al., 2016). While overexpression of PKM $\zeta$ is sufficient to both increase synaptic strength (Yao et al., 2008) and to strengthen memory (Shema et al., 2011), the isoform specificity of this ability has not been explored.

In Aplysia californica, PKM isoforms from different families are important for the maintenance of distinct forms of synaptic plasticity. The conventional, novel, and atypical PKM families are represented by PKM Apl I, PKM Apl II, and PKM Apl III, respectively (Sossin, 2007; Bougie et al., 2009). Dominant-negative (DN)-PKMs have greatly decreased kinase activity but fold correctly and participate in protein-protein interactions (Cameron et al., 2009; Bougie et al., 2012) that should allow the catalytically inactive PKMs to replace PKMs at critical sites required for maintaining increases in synaptic strength. Experiments using DNPKMs suggested that, in the postsynaptic neuron, PKM Apl I is required for the maintenance of persistent nonassociative longterm facilitation (LTF) (a cellular analog of sensitization induced by five spaced applications of 5-HT on two consecutive days), whereas PKM Apl III is required for the maintenance of persistent associative LTF (a cellular analog of classical conditioning induced by coupling firing of the sensory neuron with one application of 5-HT on 2 consecutive days) (Hu et al., 2017a,b). Because persistent associative and nonassociative LTF can coexist in one postsynaptic cell (Hu et al., 2017b), the cell must have some way of targeting distinct PKM isoforms to different synapses that underlie these forms of LTF. We propose stabilization by kidney and brain expressed adaptor protein (KIBRA) as a mechanism through which PKM isoform specificity is achieved.

KIBRA colocalizes with and stabilizes the mammalian PKM $\zeta$ (Yoshihama et al., 2009; Vogt-Eisele et al., 2014), regulates AMPA receptor trafficking, and is required for late LTP (Makuch et al., 2011; Heitz et al., 2016). KIBRA is also required for some forms of memory in rodents (Makuch et al., 2011; Vogt-Eisele et al., 2014; Heitz et al., 2016) and has been implicated in human episodic memory (Papassotiropoulos et al., 2006; Milnik et al., 2012). In Aplysia, KIBRA stabilizes overexpressed PKM Apl II and PKM Apl III, but not PKM Apl I (Hu et al., 2017b). KIBRA$\mathrm{AAA}$, in which the three residues found to be essential for PKM $\zeta$ binding were mutated (Vogt-Eisele et al., 2014), did not stabilize PKM Apl III (Hu et al., 2017b; Hastings et al., 2018) and erased the forms of LTF blocked by DN-PKM Apl III (Hu et al., 2017b), but did stabilize PKM Apl I and did not erase the forms of LTF blocked by DN-PKM Apl I. Do other isoforms of KIBRA stabilize specific PKMs?

Here, we explore the sufficiency of PKMs to increase synaptic strength and the role of KIBRA in determining isoform specificity of PKMs. Overexpression of PKMs in the motor neuron, but not the sensory neuron, increased synaptic strength. Unlike PKMs, KIBRA is not sufficient to increase synaptic strength when overexpressed in the motor neuron. DN-PKMs competed for isoform-specific stabilization by KIBRA and alternatively spliced forms of KIBRA stabilized specific isoforms of PKM based on the sequence in their $\alpha$-helix "handle" domain. Our results suggest a model whereby isoform specificity of PKMs is explained by differential stabilization by KIBRA isoforms.

\section{Materials and Methods}

Aplysia cell culture. Hermaphrodite A. californica from Miami Aplysia Resource Facility or from Alacrity Marine Biological Services (Redondo Beach, CA) were anesthetized via injection of $50-60 \mathrm{ml}$ of $400 \mathrm{~mm}$ isotonic $\mathrm{MgCl}_{2}$, and abdominal and/or pleuropedal ganglia were removed. Ganglia were digested at $19^{\circ} \mathrm{C}$ in $\mathrm{L} 15$ media containing $10 \mathrm{mg} / \mathrm{ml}$ Dispase
II (Roche Diagnostics) for $18-19 \mathrm{~h}$ or for experiments from animals from Alacrity at $35^{\circ} \mathrm{C}$ for $2 \mathrm{~h}$. L15 medium (Sigma-Aldrich) was supplemented with $0.2 \mathrm{M} \mathrm{NaCl}, 26 \mathrm{~mm} \mathrm{MgSO}_{4}, 35 \mathrm{~mm}$ dextrose, $27 \mathrm{~mm} \mathrm{MgCl}_{2}$, $4.7 \mathrm{~mm} \mathrm{KCl}, 2 \mathrm{~mm} \mathrm{NaHCO}, 9.7 \mathrm{~mm} \mathrm{CaCl}_{2}$, and $15 \mathrm{~mm}$ HEPES, with $\mathrm{pH}$ 7.4. Glass-bottom culture dishes were coated with $0.05 \%$ poly-L-lysine for $1-2 \mathrm{~h}$ and washed with $\mathrm{ddH}_{2} \mathrm{O}$ before use. Sensory neurons and LFS motor neurons were isolated from pleural and abdominal ganglia, respectively, that were dissected from adult Aplysia (60-100 g), and L7 motor neurons were isolated from the abdominal ganglia of juvenile animals ( $2 \mathrm{~g}$ ). Neurons were cultured in 50\% hemolymph/50\% L15 media supplemented with L-glutamine. For electrophysiology experiments, motor neurons were removed from the abdominal ganglia and allowed to adhere to the culture dish for $1-24 \mathrm{~h}$ before pairing with a sensory neuron from pleural ganglia as previously described (Zhao et al., 2006). Each coculture comprised a single presynaptic sensory neuron paired with a single postsynaptic motor neuron (either an LFS or an L7 neuron). Cells were incubated for $48 \mathrm{~h}$ at $19^{\circ} \mathrm{C}$ (SN-LFS) to allow time for them to adhere to the dish before injection or for $96 \mathrm{~h}$ at $19^{\circ} \mathrm{C}$ (SN-L7) to allow time for the formation of stable synapses (Hu and Schacher, 2015; Hu et al., 2017a,b). All plating, injections, and electrophysiology experiments were performed at $19^{\circ} \mathrm{C}$, except for experiments using animals from Alacrity, which were performed at room temperature.

Plasmid constructs and microinjection. All constructs were made in the pNEX3 vector (Kaang, 1996); and for all vector experiments, pNEX3 plasmid was used. All PKM constructs were made as fusion proteins with monomeric red fluorescent protein (mRFP), whereas PKC Apl I was a fusion protein with enhanced green fluorescent protein (eGFP). The mRFP or eGFP has been removed from the construct names for clarity. DN constructs (DN-PKM Apl I, DN-PKM Apl II, and DN-PKM Apl III) and PKCs/PKMS used for overexpression and stabilization studies (PKC Apl I, PKM Apl I, PKM Apl II, and PKM Apl III) were previously described (Bougie et al., 2012; Farah et al., 2017; Hu et al., 2017a). The new DN-PKM Apl III K-R was generated by cutting out this region from the plasmid encoding PKC Apl III K-R (Bougie et al., 2012) with AarI and SalI and inserting into the plasmid encoding PKM Apl III plasmid at the same sites. For the chimeras, GBLOCKS (Integrated DNA Technologies, IO) were purchased with the PKM Apl III sequences [carboxy terminus (CT) or handle] replaced by PKM Apl I sequences. These were then cut out with either BsmBI and $\mathrm{KpnI}(\mathrm{CT})$ or SalI and KpnI (handle) and inserted into the plasmid encoding PKM Apl III at the same sites. The chimeras were sequenced for confirmation. Plasmids encoding KIBRA and KIBRA-AAA were previously described (Hu et al., 2017b) and are not fusion proteins with a fluorescent protein. The KIBRA splice form, KIBRA SPL, was generated similarly to KIBRA but from a separate PCR clone that fortuitously encoded the splice form (Hu et al., 2017b). For stabilization experiments, molar equivalent levels of plasmids encoding KIBRA/KIBRA SPL/KIBRA-AAA and the PKMs/DN-PKMs were used. A solution containing the desired constructs $(\max 0.4 \mu \mathrm{g} / \mu \mathrm{l}$ of DNA in ultrapure water) with $0.2 \%$ fast green were microinjected into the nuclei of neurons using back-filled glass micropipettes. A short pressure pulse was delivered to release plasmid solution into nucleus. KIBRA and eGFP were injected into the LFS neuron of sensorimotor cocultures for intermediate facilitation (ITF) experiments. KIBRA was coexpressed with an eGFP construct so that expression could be confirmed by detecting eGFP fluorescence. Stabilization experiments were performed by injecting constructs into isolated sensory neurons. For PKM overexpression assays in sensorimotor cocultures, the constructs were injected after monitoring EPSP amplitudes on day 0. Injection into L7 motor neurons was performed as previously described (Hu et al., 2017b). Cultures were incubated at $19^{\circ} \mathrm{C}$ for $24 \mathrm{~h}$ to allow sufficient time for expression of injected plasmids before imaging.

Stabilization experiments. To measure stabilization by KIBRA, we express mRFP-tagged PKMs in the sensory neuron in the presence or absence of KIBRA and measure the intensity of mRFP in processes (to avoid red pigment granules in the cell soma that interfere with the measurement) normalized to the expression of eGFP (the plasmid encoding eGFP is coinjected with plasmid-expressing mRFP-tagged PKMs). A blind observer chooses one ROI per neuron representing a sensory neuron neurite of intermediate thickness for the measurement. The level of 
expression is measured at $24 \mathrm{~h}$ after injection, and low levels of plasmids are used to minimize toxicity and to enable expression of the plasmidexpressing KIBRA at equimolar levels to the PKMs.

Electrophysiology. For sensorimotor cocultures containing L7 motor neuron, the amplitude of the EPSP was recorded as previously described (Hu et al., 2017b). The assignment of any culture to control and experimental groups at the start of each experiment on day 4 in culture was to ensure that there were no significant differences in the synaptic strength between the groups before treatments. Sensorimotor cocultures containing LFS motor neurons were incubated in culture media at room temperature for $24 \mathrm{~h}$ following microinjection to allow sufficient time for expression of injected plasmids before electrophysiological recordings. Before recording, culture media was replaced with a recording saline (in $\mathrm{mM}$ ) as follows: $460 \mathrm{NaCl}, 55 \mathrm{MgCl}_{2}, 10 \mathrm{CaCl}_{2}, 10 \mathrm{KCl}, 10 \mathrm{D}$-glucose, and 10 HEPES, pH 7.6. Membrane potentials were recorded and controlled in current-clamp mode with sharp intracellular electrodes attached to an Axoclamp 2B amplifier (Molecular Devices). Microelectrodes (15-30 $\mathrm{M} \Omega$ ) were backfilled with $2 \mathrm{~m}$ potassium acetate and bridge-balanced before and after membrane penetration. The postsynaptic cell was impaled first, so that if entry into the presynaptic cell resulted in generation of an action potential, the resultant postsynaptic potential (PSP) would be recorded. Injection of hyperpolarizing current was used to keep both presynaptic and postsynaptic neurons at $-80 \mathrm{mV}$ during recording. Postsynaptic input resistance was measured with $500 \mathrm{~ms},-0.5 \mathrm{nA}$ pulses. For ITF experiments, electrodes were removed from cells after initial recording, and 5-HT (10 $\mu \mathrm{M})$ was added for 10 min before being washed out with $25 \mathrm{ml}$ recording saline solution. Cultures were left at $19^{\circ} \mathrm{C}$ for $2 \mathrm{~h}$, at which time the second recording was performed. Initial PSP rise rate was measured as previously described (Dunn and Sossin, 2013). For PKM overexpression experiments (see Fig. 1), EPSP amplitudes were measured before microinjection (day $0=96 \mathrm{~h}$ after cell plating to insure stable synapses), and again on days 1, 3, and 5 as described previously (Bougie et al., 2012; Hu et al., 2017a). The kinetics of homosynaptic depression (HSD) were monitored on day 1 by homosynaptic stimulation, stimulating the sensory neuron with one action potential every $20 \mathrm{~s}$, 6 stimuli in total (Hu et al., 2017b). The motor neuron was maintained at $-80 \mathrm{mV}$ during the stimulation. The initial EPSP amplitude evoked by the first stimulus was normalized as $100 \%$.

Immunocytochemistry. Cultured cells were fixed with $4 \%$ PFA $+30 \%$ sucrose in PBS for $30 \mathrm{~min}$ and washed with PBS. Fixed cells were permeabilized (0.1\% Triton X-100 with 30\% sucrose in PBS for $10 \mathrm{~min})$, and free aldehydes were quenched (50 mM ammonium chloride for $10 \mathrm{~min}$, followed by four PBS washes). Cells were incubated with $10 \%$ normal goat serum (Sigma-Aldrich) $+0.5 \%$ Triton X-100 in PBS for 30 min to block nonspecific antibody binding. Cells were then treated with PKC Apl III antibody in blocking solution (1:5000) for $1 \mathrm{~h}$, followed by five PBS washes (10 min each). Cells were then incubated with AlexaFluor647-conjugated donkey anti-rabbit antibody in blocking solution (1:500, Invitrogen) for $1 \mathrm{~h}$ in darkness and washed with PBS as described above.

Imaging and image analysis. Images were captured by an LSM 710 (Carl Zeiss) laser confocal scanning microscope equipped with an Axiovert 100 inverted microscope (Carl Zeiss) and a 40×, NA 1.4 objective. The eGFP, mRFP, and/or A647 images were acquired sequentially. Images for the expression of PKM constructs in sensorimotor cocultures were viewed with a Nikon Diaphot microscope attached to a siliconintensified target (Dage 68; Dage-MTI) video camera. Fluorescence intensity (arbitrary units) was measured by the MicrocomputerControlled Imaging Device software package (Imaging Research). For analysis of DN-PKM and PKM stabilization, single processes for each neuron were outlined using National Institutes of Health's ImageJ. Background fluorescence was subtracted from the fluorescence values measured. The red/green or cyan/green ratio for all neurons was normalized to the average ratio seen in vector-injected neurons from the same experiment. All quantification of stabilization was done blindly.

Evolution analysis. Orthologs of KIBRA were determined using the reverse BLAST method as described below. Organisms with established genomes on NCBI were searched using BLAST, to identify proteins with homology to KIBRA. To distinguish between true orthologs and proteins with similar WW and C2 domains, these proteins were then used as a query in a BLAST search; and if they are much more homologous to other proteins with WW and C2 domains than to KIBRA, they were rejected as orthologs. Based on this, no KIBRA orthologs exist in sponge (Amphimedon queenslandia), Trichoplax (Trichoplax adhaerens), Ctenophores (Mnemiopsis), and choanoflagellate (Monosiga bervicolis; Salpingoeca rosetta). KIBRA orthologs were found in other Cnidaria (Coral, Styklophora postillata and Acropora digitefara; jellyfish, Hydra vulgaris), but the strongest homology was to Anemone, Nemostella vectensis, and this Cnidarian was used in the comparison studies.

Behavioral training. Adult Aplysia (80-120 g) were given $1 \mathrm{~d}$ of longterm sensitization training similar to previously described protocols (Cai et al., 2011; Chen et al., 2014). The training consisted of 5 bouts of tail shocks delivered via implanted platinum wires. Each bout comprised three trains (train duration $=1 \mathrm{~s}$ ) of shocks $(10 \mathrm{~ms}$ pulse duration, $40 \mathrm{~Hz}$, $120 \mathrm{~V}$ ) spaced $2 \mathrm{~s}$ apart; the bouts of shocks were separated by $20 \mathrm{~min}$ intervals. Before the shocks, each animal was given three pretests, spaced 10 min apart, during which a brief, relatively weak tactile stimulus was applied to its siphon; the duration of the resulting siphon-withdrawal response provided an index of sensitization. Forty-eight hours after the sensitization training, each snail was given a single test stimulus, and its siphon-withdrawal response measured. Another group of snails were treated identically to the trained group, except that the tail shocks on day 1 were withheld. Animals that received the shock training exhibited a significantly prolonged siphon-withdrawal response compared with animals that received only the test stimuli (Chen et al., 2014). Immediately after the $48 \mathrm{~h}$ test, the animals were anesthetized by immersion in cold $\left(4^{\circ} \mathrm{C}\right)$ seawater, and their abdominal ganglia were dissected out and prepared for qRT-PCR.

$q R T-P C R$. Total RNA was extracted from abdominal ganglia of trained and control (test alone) Aplysia using Trizol reagent following the manufacturer's instructions. cDNA was synthesized from $2 \mu \mathrm{g}$ total RNA using qScript cDNA SuperMix (QuantaBio) to a final concentration of $100 \mathrm{ng} / \mu \mathrm{l}$. KIBRA primer pair sequences were used: forward TGG AGA AAC TCC TGC AAG GCC A; reverse ATT GCT GGC GTC TGC TAA CTC. qRT-PCR was performed using the ABI PRISM 7900 sequencing detection system (Applied Biosystems) with SYBR Green master mix under the following conditions: initial denaturation at $95^{\circ} \mathrm{C}$ for $10 \mathrm{~min}$, followed by 40 cycles of $95^{\circ} \mathrm{C}$ for $15 \mathrm{~s}$ and $65^{\circ} \mathrm{C}$ for $30 \mathrm{~s}, 72^{\circ} 30 \mathrm{~s} ; 5 \mu \mathrm{l}$ SYBR, $3 \mu \mathrm{l} \mathrm{H}_{2} \mathrm{O}, 1 \mu \mathrm{l}$ cDNA per reaction, each sample was run in triplicate. The data were quantified by the 2(-delta delta $\mathrm{C}(\mathrm{T})$ )method (Livak and Schmittgen, 2001). H4 was used as an internal control.

Statistical analysis. In all experiments, the $N$ is described as each sensory-motor neuron culture; or for stabilization experiments, each sensory neuron. An independent experiment is defined when sensory and motor neurons are obtained at the same time from the same animals and all conditions are done in parallel. For experiments with multiple measurement times, a two-way ANOVA (group $\times$ repeated measures) was used with pairwise Bonferroni post hoc tests to determine significance of individual time points. For experiments with multiple groups, a oneway ANOVA was used with Tukey post hoc tests to determine significant differences between groups. For all experiments with only two groups, Student's $t$ tests were used to determine whether the groups were different; and when multiple groups were compared this way in a single experiment, a Bonferroni correction for multiple $t$ tests was performed.

\section{Results}

\section{Postsynaptic, but not presynaptic, overexpression of PKM is sufficient to produce a long-lasting increase in synaptic strength}

In vertebrates, overexpression of $\mathrm{PKM} \zeta$ is sufficient to increase synaptic strength when expressed in the postsynaptic neuron (Yao et al., 2008). However, there have been no studies examining the isoform specificity of this effect or whether PKMs are sufficient to increase synaptic strength when overexpressed in the presynaptic neuron. Overexpression of any of the three Aplysia PKM isoforms in the postsynaptic motor neuron of Aplysia sensorimotor cocultures is sufficient to increase synaptic strength 
A Postsynaptic PKM expression

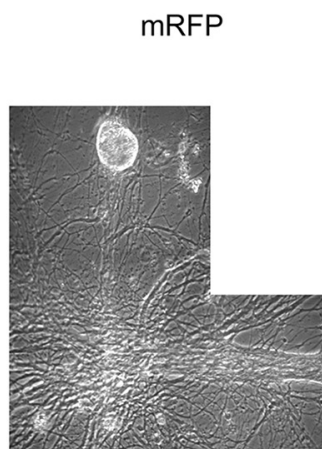

PKM Apl I

PKM Apl II

PKM Apl III
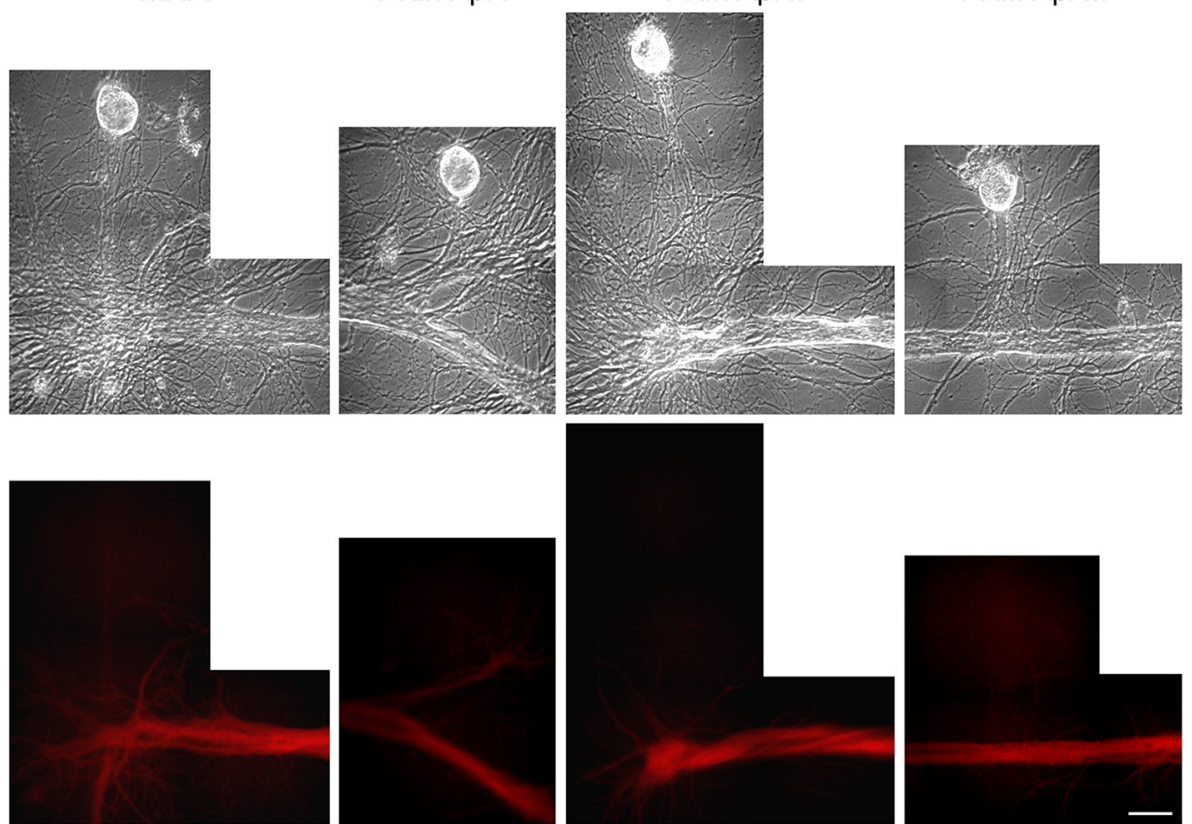

B
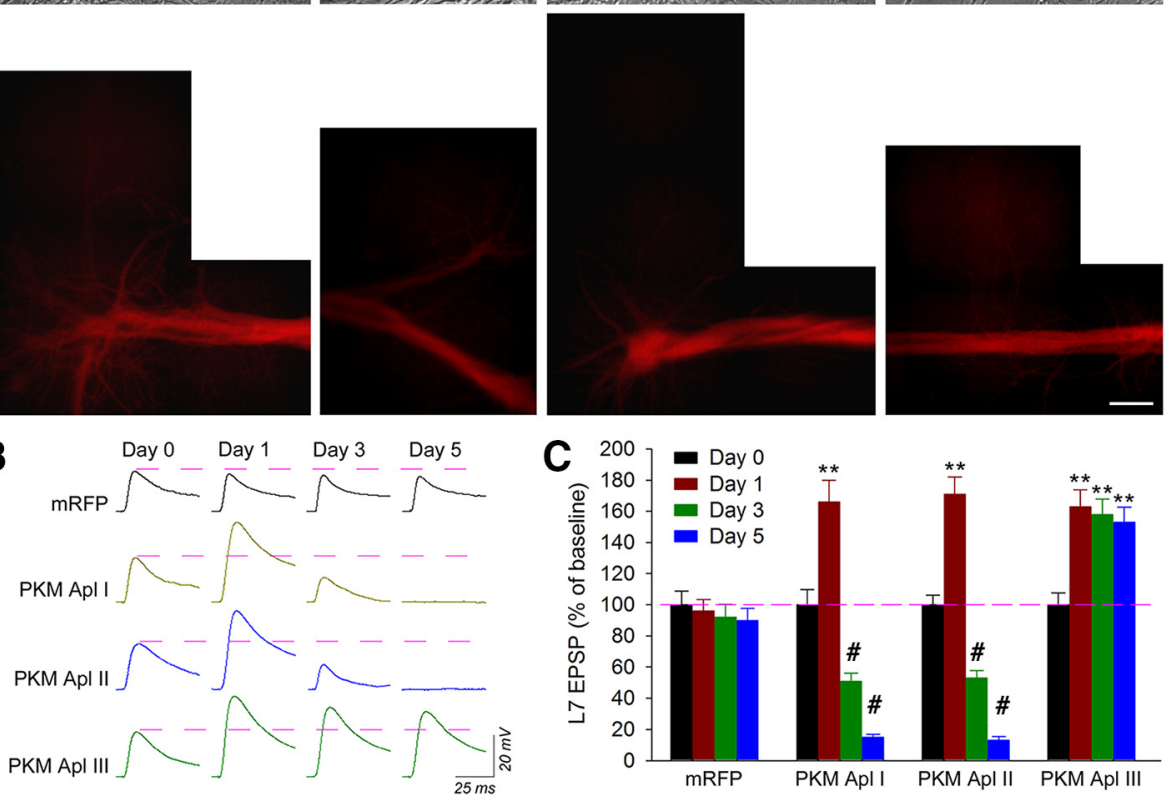

\section{Presynaptic PKM expression}
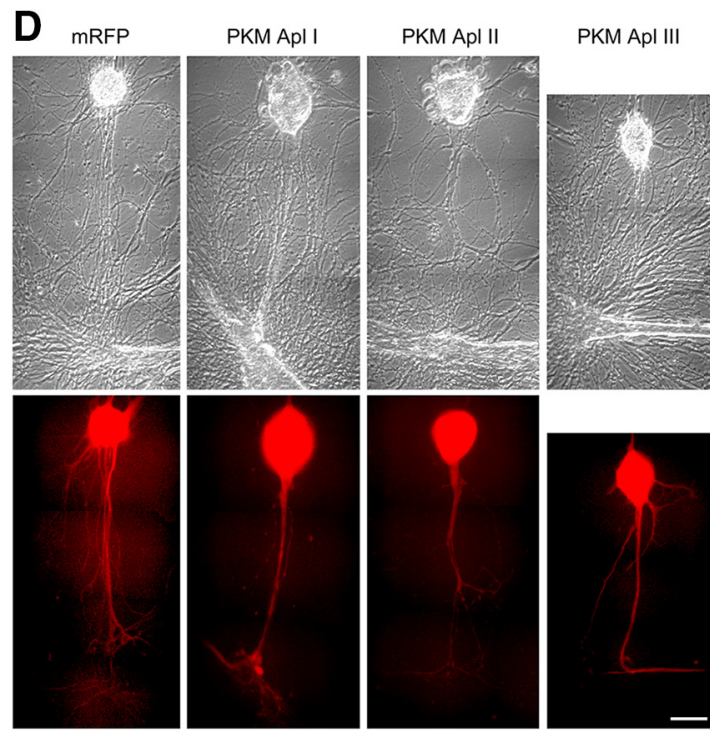

E
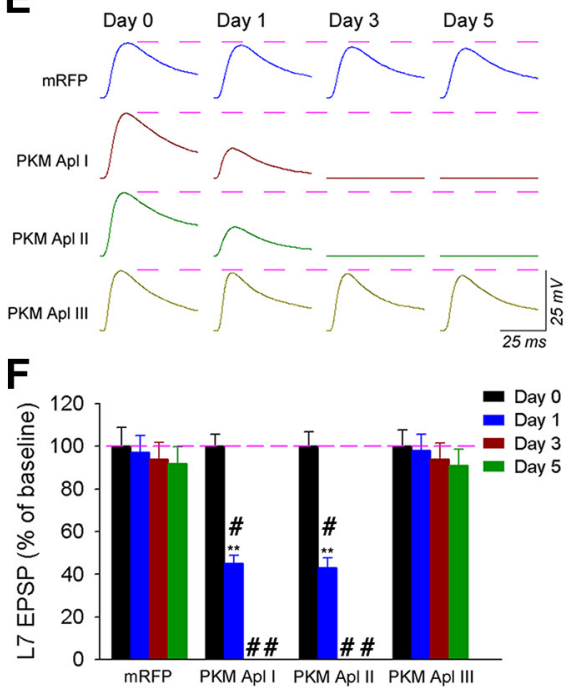

Figure 1. Presynaptic and postsynaptic overexpression of PKM isoforms produce different changes in synaptic strength. $A$, Phase contrast and epifluorescent views of cocultures $24 \mathrm{~h}$ after the indicated construct was injected into each postsynaptic neuron L7. The constructs were injected after monitoring EPSP amplitudes on day 0 ( $96 \mathrm{~h}$ after plating sensory neuron and L7). Scale bar, 25 $\mu \mathrm{m} . \mathbf{B}$, Sample EPSP traces for each treatment. Dashed lines indicate the initial EPSP amplitude. EPSPs recorded from cultures before (day 0 ) and at various times after injection of the indicated construct. Overexpression of PKM ApI III in L7 produced a significant increase in EPSP amplitude compared with control (overexpressed mRFP) and persisted to day 5. Overexpression of PKM Ap I or PKM ApI II also produced a significant increase in EPSP amplitude on day 1, but EPSP amplitude declined after day 3. C, Summary of the change in EPSP amplitudes (Figure legend continues.) 
A

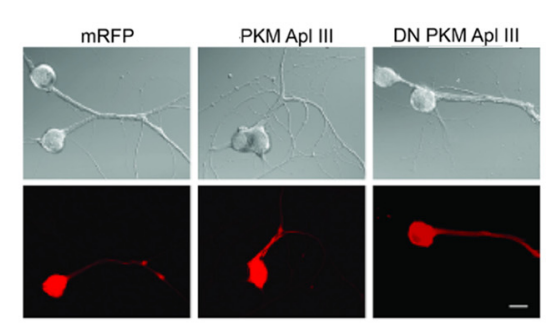

C

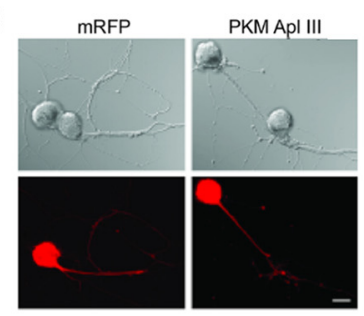

B

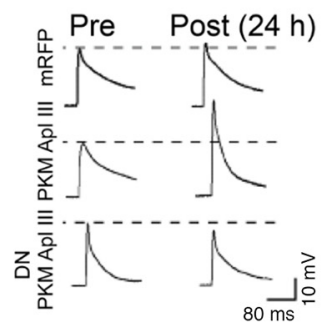

D

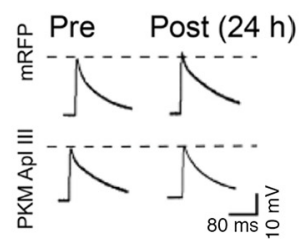

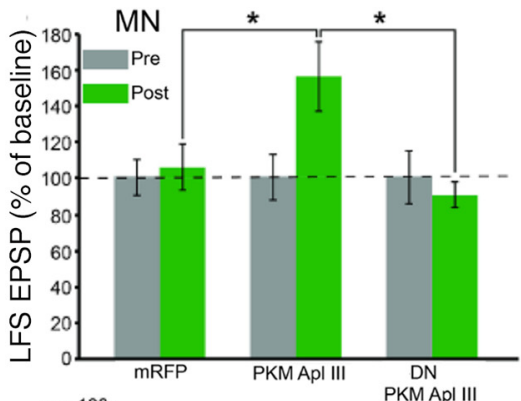

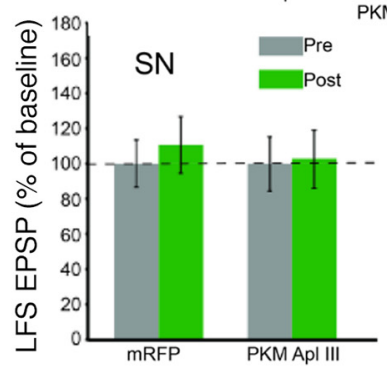

Figure 2. Overexpression of PKM Apl III in the LFS motor neurons, not in the sensory neurons, produces a long-term increase in synaptic strength. $\boldsymbol{A}$, $\boldsymbol{C}$, Phase contrast and epifluorescent images of the same view areas of LFS motor neurons $(\boldsymbol{A})$ or sensory neurons $(\boldsymbol{C})$ expressing mRFP or PKM ApI III $24 \mathrm{~h}$ after injection of constructs. The constructs were injected into the sensory or the motor neurons on day 4 of cell culturing. Scale bar, $20 \mu \mathrm{m}$. B, Overexpression of PKM ApI III, but not DN-PKM Apl III, in LFS motor neurons produces an increase in synaptic strength. Sample traces show EPSPs evoked in the LFS motor neuron before (pre) and $24 \mathrm{~h}$ after (post) control and injections of constructs in the motor neuron. The accompanying histogram is a summary of the change in the EPSP amplitudes. The mean normalized EPSP in the mRFP group ( $n=28,12$ independent experiments), PKM Apl III group ( $n=22,12$ independent experiments), and DN-PKM ApI III group ( $n=12,6$ independent experiments) was $106 \pm 13 \%, 154 \pm 19 \%$, and $90 \pm 7 \%$, respectively. A one-way ANOVA indicated that the overall group differences were significant $\left(F_{(2,59)}=4.4, p=0.02\right)$. SNK post hoc tests showed that the mean normalized EPSP in the PKM ApI III group was significantly higher than that in the mRFP and DN-PKM ApI III groups, as indicated: ${ }^{*}<<0.05$ for both comparisons. The difference between the mRFP and DN-PKM ApI III groups was not significant. D, Overexpression of PKM Apl III in sensory neurons does not affect synaptic strength. Sample traces show EPSPs evoked in the LFS motor neuron before (pre) and $24 \mathrm{~h}$ after (post) control and injections of constructs in the sensory neuron. The accompanying histogram is a summary of the change in the EPSP amplitudes. There was no effect of presynaptic overexpression of PKM ApI III. The mean normalized EPSP in the PKM ApI III group ( $n=9,6$ independent experiments) and mRFP group ( $n=9,5$ independent experiments) was $103 \pm 17 \%$ and $111 \pm 16 \%$, respectively.

\section{$\longleftarrow$}

(Figure legend continued.) (\% of baseline). A two-way ANOVA indicated a significant effect of group $\times$ repeated measures $\left(F_{(9,69)}=104.831, p<0.0001\right)$. Pairwise comparisons (Bonferroni) indicated that overexpression of PKMApI III in L7 ( $n=14)$ significantly increased EPSP amplitudes on days 1,3 , and 5 compared with mRFP $(n=8) .{ }^{* *} p<0.01$. Overexpression of PKMApI I $(n=11)$ or PKM ApI II $(n=9)$ significantly increased EPSP amplitudes on day 1 compared with mRFP (** $p<0.01$ ), but EPSP amplitudes declined after day 3 because of degeneration in $\mathrm{L} 7$ as indicated by changes in membrane resting potential (\#). 0 day 5 , only 3 of 11 cultures with overexpression of PKM Apl I had small EPSPs, the resting membrane potentials of $L 7$ in the other 8 cultures without EPSPs were close to zero, while 2 of 9 cultures with overexpression of PKM ApI II had small EPSPs and the other 7 cultures that lacked EPSPS had resting potentials close to zero. Error bars indicate SEM. Results are from seven independent experiments. $\boldsymbol{D}$, Phase contrast and epifluorescent views of cocultures $24 \mathrm{~h}$ after the indicated construct was injected into each SN. The constructs were injected after monitoring EPSP amplitudes on day 0 ( $96 \mathrm{~h}$ after plating). Scale bar, $25 \mu \mathrm{m}$. E, Sample EPSP traces for each treatment. Dashed lines indicate the synaptic baseline. EPSPs recorded from cultures before (day 0 ) and at various times after injection of the indicated construct. Overexpression of PKM ApI III had no significant impact on EPSP amplitude compared with control (mRFP overexpression). In contrast, overexpression of PKM ApI I or PKM ApI II produced a significant decline in EPSP amplitude on day 1 due to detectable neurite degeneration (\#). Viability of sensory neurons (extensive degeneration of cell body and neurites) was compromised by day 3, resulting in an absence of EPSPs. $\boldsymbol{F}$, Summary of the change in EPSP amplitudes (\% of baseline). Overexpression of PKM ApI III in SN $(n=11)$ evoked no change in EPSP amplitude compared with control $(n=7)$, whereas overexpression of PKM ApI I $(n=11)$ or PKM ApI II $(n=10)$ produced a significant decline in EPSP and subsequent death of the sensory neuron. A two-way ANOVA indicated a significant effect of group $\times$ repeated measures $\left(F_{(9,105)}=100.507, p<\right.$ 0.0001). Pairwise comparisons (Bonferroni) indicated that overexpression of PKM ApI III evoked no significant change on days 1,3 , and 5 compared with control (all $p>0.9$ ). Overexpression of PKM Apl I or PKM Apl II produced a significant decline in EPSP amplitudes on day 1 (all ${ }^{* *} p<0.01$ ). Error bars indicate SEM. Results are from six independent experiments.
$24 \mathrm{~h}$ following injection compared with overexpression of mRFP alone (Fig. 1A-C). Expression of the PKMs was highest in the cell body and major axons of L7 and decreased in intensity in the distal neurites. The range of intensity of expression for each PKM was similar in the distal neurites and did not correlate with the observed changes in synaptic strength or toxicity over time. Continued expression of PKM Apl I or PKM Apl II is toxic, whereas overexpression of PKM Apl III can maintain increased synaptic strength for a prolonged period (Fig. $1 A-C$ ). By day 5 , only 3 of 11 cultures overexpressing PKM Apl I had small EPSPs, whereas the resting membrane potentials of $\mathrm{L} 7$ in the other 8 cultures without EPSPs were close to zero. Only 2 of 9 cultures overexpressing PKM Apl II had small EPSPs, and the other 7 cultures that lacked EPSPs had resting potentials close to zero. Morphologically, however, all of the L7 neurons overexpressing either PKM Apl I or PKM Apl II showed little, if any, observable degeneration even on day 5. In contrast, none of the PKMs increased synaptic strength $24 \mathrm{~h}$ following injection when expressed in the sensory neuron (Fig. $1 D-F$ ). Similar to the motor neuron, overexpression of PKM Apl I and PKM Apl II in the sensory neuron was toxic, but PKM Apl III was not (Fig. $1 D-F$ ). After injection of PKM Apl I and PKM Apl II, some neurite degeneration was detected on day 1 and was extensive by day 3, resulting in the absence of EPSPs. All SN cell bodies expressing either PKM Apl I or PKM Apl II degenerated by day 5 .

These results were obtained using the postsynaptic neuron L7 (gill withdrawal motor neuron), but similar results were seen for PKM Apl III in the presynaptic and postsynaptic neuron when LFS neurons (siphon withdrawal motor neurons) were used as the postsynaptic neurons (Fig. 2). 

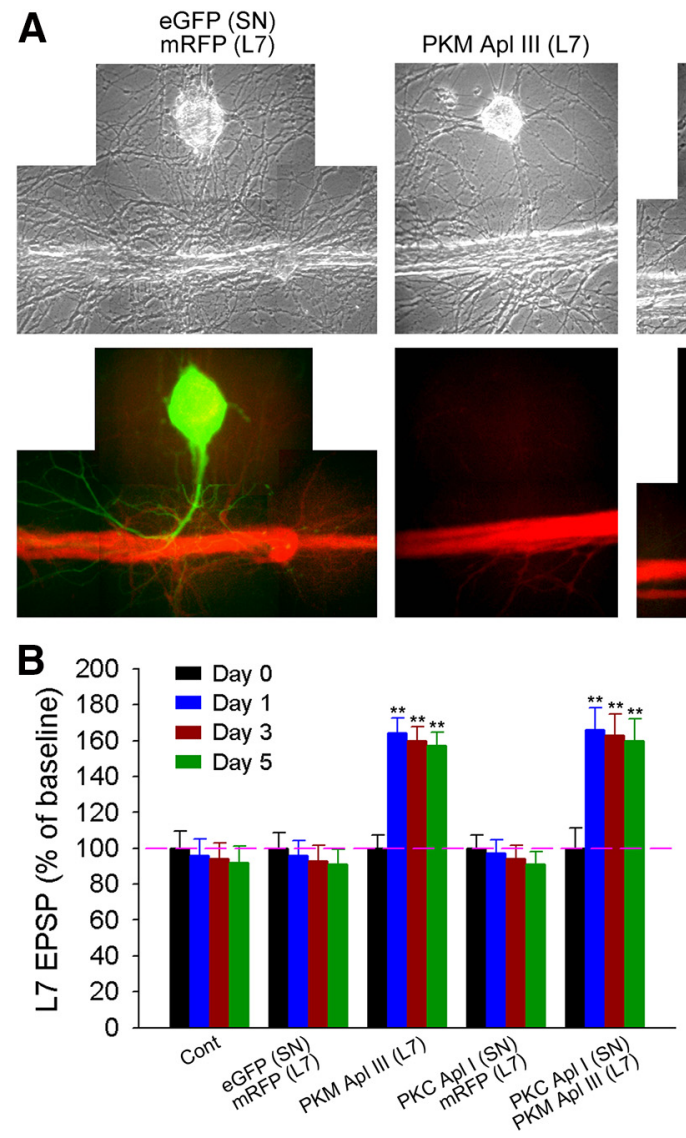
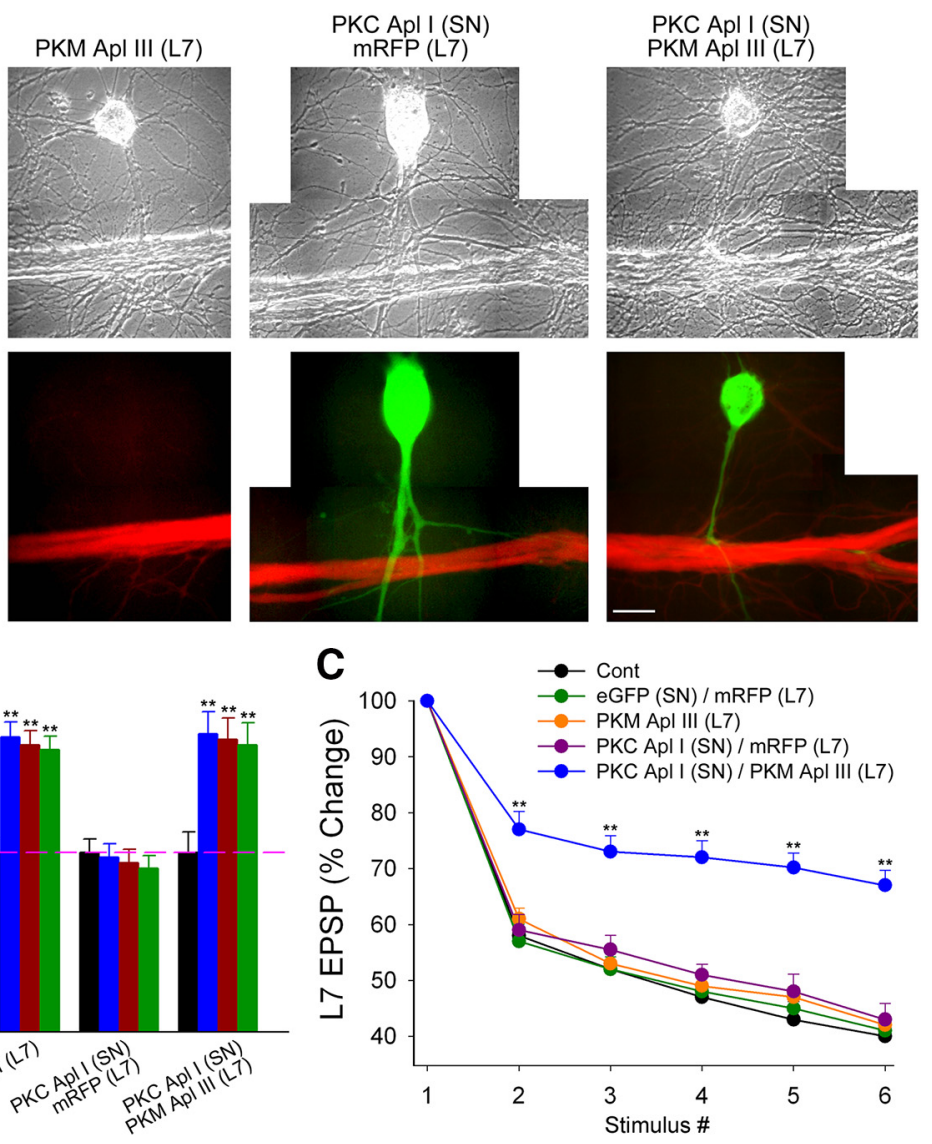

Figure 3. The attenuation of HSD requires kinase activity in both presynaptic and postsynaptic neurons. $A$, Phase contrast and epifluorescent views of cocultures $24 \mathrm{~h}$ after the indicated construct injection in SN and L7. Scale bar, $25 \mu \mathrm{m}$. B. Summary of the change in EPSP amplitudes (\% of baseline). Overexpression of PKM Apl III alone in L7 ( $n=12$ ) or co-overexpression of PKC ApI I in SN and PKM ApI III in L7 ( $n=10)$ produced a persistent increase in the synaptic strength. The other overexpressions failed to increase synaptic strength and were not significantly different from control. A two-way ANOVA indicated a significant effect of group $\times$ repeated measures $\left(F_{(12,108)}=65.656, p<0.0001\right)$. Pairwise comparisons (Bonferroni) indicated that overexpression of either PKM Apl III alone in L7 or both PKC ApI I in SN and PKM ApI III in L7 evoked significant facilitation on days 1, 3, and 5 compared with control ( $n=6$ ), c0-overexpression of eGFP in SN and mRFP in L7 ( $n=7$ ), or co-overexpression of PKC Apl I in SN and mRFP in L7 $(n=6) .{ }^{* *} p<0.01$. Error bars indicate SEM. C, The kinetics of HSD after co-overexpression of PKC Apl I in SN and PKM ApI III in L7 was attenuated. Compared with control, only co-overexpression of PKC Apl I in SN and PKM Apl III in L7 evoked an attenuated kinetics of HSD that is observed at sensorimotor synapses expressing persistent associative LTF, whereas other overexpressions failed to alter HSD kinetics. A two-way ANOVA indicated a significant effect of group $\times$ repeated measures on the kinetics of HSD $\left(F_{(20,180)}\right.$ $=15.025 ; p<0.0001$ ). Pairwise comparisons (Bonferroni) indicated that the kinetics of HSD only with co-overexpression of PKC Apl I in SN and PKM ApI III in L7 was significantly different from that for control. ${ }^{* *} p<0.01$. Error bars indicate SEM. Results are from five independent experiments.

A

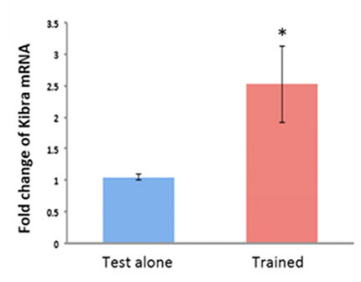

B

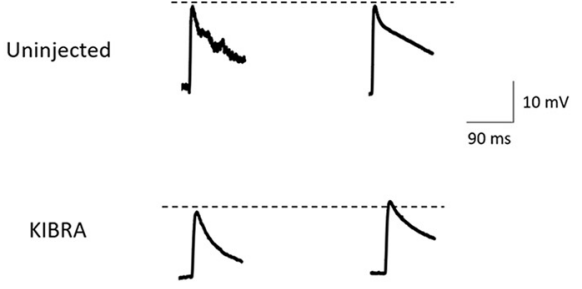

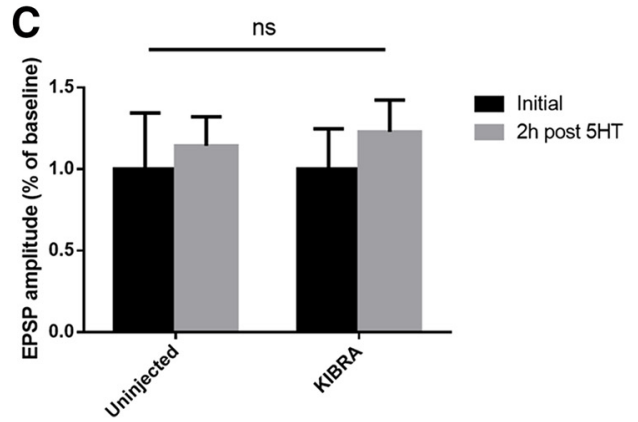

Figure 4. KIBRA overexpression is not sufficient to prolong PKM ApI III-dependent ITF. A, qRT-PCR results comparing levels of KIBRA mRNA from cDNA generated from control abdominal ganglia (8 animals) or abdominal ganglia from sensitized animals $48 \mathrm{~h}$ after training (7 animals). Sensitization experiments were performed as described previously (Cai et al., 2011$)$. Results are mean \pm SEM. ${ }^{*} p<0.05$ (one-tailed $t$ test). $\boldsymbol{B}$, Sample traces before and $2 \mathrm{~h}$ after $10 \mathrm{~min} 10 \mu \mathrm{m} 5$-HT treatment in sensorimotor cocultures using LFS as the postsynaptic motor neuron, overexpressing KIBRA (with eGFP to confirm expression) in the postsynaptic neuron or uninjected controls. Calibration: $90 \mathrm{mV} / 10 \mathrm{~ms}$. C, Summary of the change in EPSP amplitude $2 \mathrm{~h}$ after 5 -HT treatment in postsynaptic neurons expressing KIBRA $(n=8)$ and uninjected controls $(n=5)$. The change in PSP at $2 \mathrm{~h}$ was similar between groups via unpaired $t$ test (not significant, $p=0.4380)$. Initial EPSP amplitude was not significantly different between groups (one-way unpaired $t$ test; not significant, $p=0.7613$ ). Initial postsynaptic input resistance and the change in postsynaptic input resistance were similar between groups $(180.0 \pm 27.4 \mathrm{~m} \Omega$ uninjected and $177.9 \pm 35.6 \mathrm{~m} \Omega$ eGFP + KIBRA, $p=0.9661$ that changed to $92.4 \pm 6.0 \%$ and $78.6 \pm 6.2 \%$, respectively, at $2 \mathrm{~h}, p=0.1448$ compared with unpaired $t$ tests). 
Aplysia sensory neurons undergo remarkable synaptic depression after a sequence of low-frequency action potentials ( 6 action potentials at $0.05 \mathrm{~Hz}$ in our experiments) that is termed HSD and is mediated by a decrease in presynaptic release (Armitage and Siegelbaum, 1998). After 2 $\mathrm{d}$ of training, persistent associative LTF, but not persistent nonassociative LTF, is also accompanied by an attenuated form of HSD (Hu and Schacher 2014, 2015), suggestive of presynaptic changes in the release machinery (Wan et al., 2012). Nevertheless, DN-PKM Apl III expressed in the motor neuron erases persistent associative LTF and the maintenance of the attenuated form of HSD ( $\mathrm{Hu}$ et al., 2017a). However, overexpression of PKM Apl III in the postsynaptic neuron, while sufficient to induce and maintain an increase in synaptic strength, is not sufficient to induce the expression of the attenuated form of HSD measured $24 \mathrm{~h}$ after expression (Fig. $3 A-C$ ). DN-PKM Apl II expressed in the sensory neuron erases persistent associative LTF and the attenuation of HSD (Hu et al., 2017a); but since PKM Apl II expression in the sensory neuron is toxic (Fig. $1 D-F$ ), we determined the effect of overexpression of PKC Apl I in the sensory neuron. DNPKC Apl I expressed in the SN also reversed the attenuation of HSD (Hu et al., 2017a). While overexpression of PKC Apl I was not sufficient to increase synaptic strength or to induce the expression of attenuated HSD on its own, it was not toxic (Fig. 3A-C). Expression of both PKC Apl I in the sensory neuron and PKM Apl III in the motor neuron produced both persistent LTF and attenuation of HSD (Fig. $3 A-C)$. Thus, the induction of both forms of plasticity observed at synapses expressing persistent associative LTF produced by repeated paired stimulation (synapse facilitation and attenuated HSD) requires increased kinase activity in both the presynaptic and postsynaptic neuron.

\section{KIBRA overexpression is not sufficient to prolong ITF}

Forms of facilitation that last longer than short-term facilitation but do not require transcription are termed ITF in Aplysia. There are different forms of ITF based on the stimulation, but massed ITF ( $\mathrm{m}$-ITF) is of particular interest as it requires cleavage of PKC Apl III to PKM Apl III (Bougie et al., 2012; Farah et al., 2017). Despite the requirement for a PKM, this form of facilitation is transient (Sutton et al., 2002; McCamphill et al., 2015). One hypothesis is that PKMs are made but not stabilized in the absence of KIBRA; thus, transcriptional upregulation of KIBRA could be important for the prolongation of PKM activity and increases in synaptic strength. Supporting this hypothesis, KIBRA stabilizes PKM Apl III (Hu et al., 2017b); and $48 \mathrm{~h}$ after induction of longterm behavioral sensitization in Aplysia, performed as described previously (Cai et al., 2011), there was an upregulation of KIBRA indicate SEM.

\section{B}

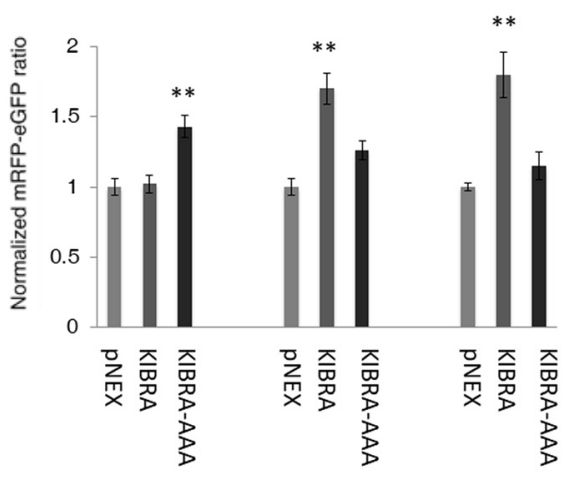

DN PKM AplI DN PKM Apl II DN PKM Apl III

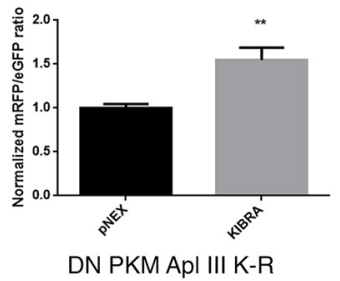

DN PKM Apl III K-R

Figure 5. KIBRA stabilizes inactive PKMs in isolated Aplysia sensory neurons. A, Representative images of eGFP and DN-PKM Apl (D392-A) (top), DN-PKM Apl II (D444-A) (middle), and DN-PKM ApI III (D526-A) (bottom) with vector (pNEX3), KIBRA, or KIBRAof mRFP-tagged DN-PKMs is quantified as the average mRFP/eGFP ratio normalized within each experiment to the average mRFP/eGFP ratio of the pNEX group. KIBRA (AAA) stabilizes DN-PKM ApI I, whereas KIBRA stabilizes DN-PKM ApI II and DN-PKM ApI III. ANOVAs were performed separately for DN-PKM ApI I (pNEX, $n=39$ neurons; KIBRA, $n=66$ neurons; KIBRA RSR-AAA $n=70$ neurons; neurons from four independent experiments). One-way ANOVA $\left(F_{(2.172)}=12.2\right): p<0.001$ with Tukey's post hoc test. . versus all other groups. DN-PKM Apl III (pNEX, $n=28$ neurons; KIBRA, $n=36$ neurons; KIBRA RSR-AAA, $_{n} n=24$ neurons; neurons 作 all other groups. C, Representative images of eGFP and mRFP-tagged PKM ApI III K-R with either pNEX or KIBRA in neurites of Aplysia sensory neurons $24 \mathrm{~h}$ after injection. D, Stabilization of PKM Apl III K-R is quantified as the average mRFP/eGFP ons; pNEX, $n=24$ neurons; neurons from three independent experiments). ${ }^{* *} p<0.01$, unpaired Student's $t$ test. Error bars

mRNA as measured by qRT-PCR (Fig. 4A). To determine whether increased levels of KIBRA are sufficient to maintain memories by stabilizing PKMs, we induced $\mathrm{m}$-ITF in neurons expressing KIBRA. KIBRA did not, however, increase m-ITF at $2 \mathrm{~h}$ after induction, a time in which $\mathrm{m}$-ITF has decayed to baseline (Sutton et al., 2002; McCamphill et al., 2015) (Fig. 4B). This was not due to an occlusion by KIBRA as there was no increase in basal synaptic strength in the KIBRA-expressing neurons (control: $35.99 \pm 12.8 \mathrm{mV}$; KIBRA: $30.31 \pm 7.4 \mathrm{mV}$; unpaired $t$ test $p=0.6919)$.

\section{Competition for stabilization by KIBRA accounts for isoform-specific effects of DN-PKMs}

Distinct DN-PKMs expressed in the postsynaptic motor neuron erase either persistent associative or persistent nonassociative LTF, even when the two forms of LTF are simultaneously expressed in the same postsynaptic neuron (Hu et al., 2017b). Similarly, the two forms of LTF have distinct stimuli that activate reconsolidation, and only the stimuli that induced persistent associative LTF lead to attenuation of HSD (Hu et al., 2017a,b). 
Thus, there are different mechanisms underlying the expression of the distinct types of LTF. However, overexpression of any PKM in the motor neuron is sufficient to induce an increase in synaptic strength, making it difficult to understand why distinct PKMs are required for maintenance of persistent associative and nonassociative LTF (Hu et al., 2017b). One possibility is that, at endogenous levels of PKMs, isoform-specific stabilization at synapses by the adaptor protein KIBRA is required. Aplysia KIBRA stabilizes PKM Apl II and PKM Apl III, but not PKM Apl I (Hu et al., 2017b) in an assay where levels of mRFP-PKMs in sensory neurons (standardized to an EGFP control) are measured in the presence or absence of KIBRA after $24 \mathrm{~h}$ of expression. Similarly, DN-PKM Apl II and DN-PKM Apl III erase associative LTF when expressed in the motor neuron, as does a form of KIBRA (KIBRA-AAA) that does not stabilize PKM Apl III (Hu et al., 2017b). Thus, DN-PKMs may work by competing with endogenously produced PKMs for KIBRA-mediated stabilization. One issue with this proposal is the report that vertebrate KIBRA does not bind to catalytically inactive PKMs (Vogt-Eisele et al., 2014), and the DN-PKMs used in this study are catalytically inactive. However, in Aplysia neurons, we found that KIBRA stabilizes the DN-PKMs (Fig. 5A,B). The pattern of stabilization between KIBRA and KIBRA-AAA was similar to that seen with catalytically active PKMs (Hu et al., 2017b): KIBRA stabilizes DN-PKM Apl III and DN-PKM Apl II, whereas KIBRA-AAA stabilizes DNPKM Apl I. These DN-PKMs were generated by an aspartate to alanine (D-A) mutation at the catalytic aspartic acid (Cameron et al., 2009). This mutation allows for correct folding of PKC/PKM as measured by correct priming phosphorylation (Cameron et al., 2009; Bougie et al., 2012); by contrast, the kinase dead mutant used in Vogt-Eisele et al. (2014), produced through the mutation of a lysine in the ATP binding pocket, results in a PKC that lacks priming phosphorylation (Cameron et al., 2009). However, PKM Apl III with a similar lysine mutation (K279R, numbering from PKC Apl III) that also does not receive priming phosphorylation (Bougie et al., 2012) was also stabilized by KIBRA (Fig. 5C,D). Thus, in Aplysia, PKMs do not need to be catalytically active or have priming phosphorylation in order for KIBRA-mediated stabilization to occur.

KIBRA-AAA stabilizes PKM Apl II, but overexpression of KIBRA-AAA in the postsynaptic motor neuron results in a reversal of associative LTF (Hu et al., 2017b), suggesting that PKM Apl II is not involved in associative LTF. However, DN-PKM Apl II was able to reverse associative LTF (Hu et al., 2017a). One explanation for this discrepancy is that the overexpressed DN-PKM Apl II is competing with endogenous PKM Apl III for KIBRA binding, resulting in a decrease in synaptic strength due to the destabilization of endogenous PKM Apl III. To test this specifically, we overexpressed PKM Apl III along with KIBRA or empty pNEX control and looked at whether concurrent overexpression of DN-PKM Apl II affected KIBRA-mediated PKM Apl III stabilization. We also overexpressed DN-PKM Apl I to confirm that this competition for KIBRA binding is specific for DN-PKM Apl II, as DN-PKM Apl I is not stabilized by KIBRA (Fig. 5). As both the DN constructs and the PKM Apl III construct were tagged with mRFP, we quantified PKM Apl III levels using a PKC Apl III C-terminal antibody (Bougie et al., 2009). Overexpression of DN-PKM Apl I did not affect KIBRA's stabilization of PKM Apl III, whereas the stabilization of PKM Apl III in the presence of DN-PKM Apl II was significantly reduced and, indeed, not significantly different from the vector control (Fig. 6A,B). This demonstrates that DN-PKM Apl II is able to interfere with

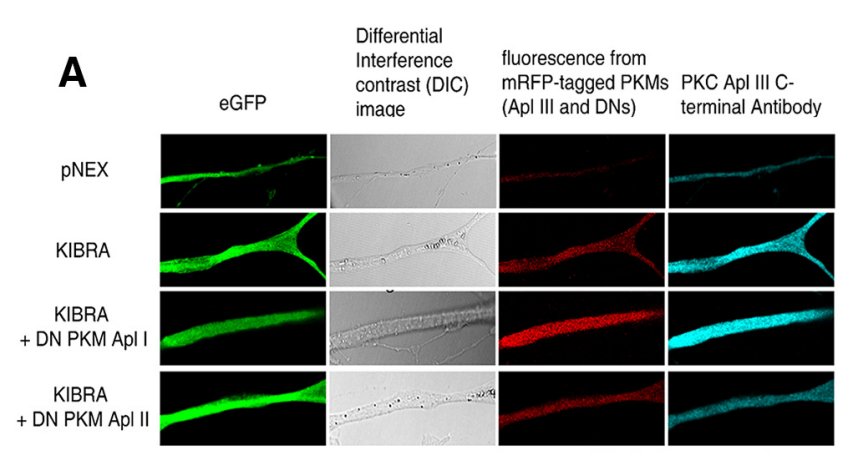

B

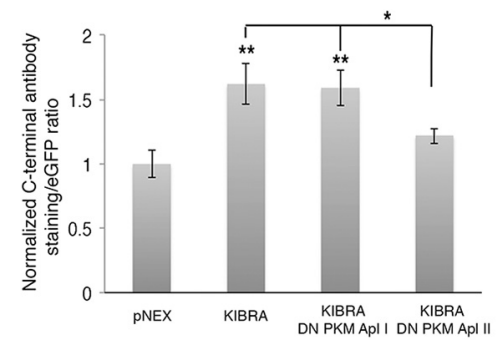

Figure 6. Overexpression of DN-PKM ApI II interferes with KIBRA-mediated stabilization of overexpressed PKM ApI III. $\boldsymbol{A}$, Representative images of cultured Aplysia sensory neuron neurites coexpressing eGFP, mRFP-tagged PKM ApI III, mRFP-tagged DN-PKM ApI I, or DN-PKM ApI II, and either KIBRA or empty vector (pNEX3). Cultures were fixed and stained with PKC ApI III C-terminal antibody (cyan) before imaging (PKC Apl III and PKM Apl III have the same (-terminal). B, PKM ApI III stabilization is quantified as the ratio between eGFP fluorescence and PKC Apl III C-terminal antibody staining (cyan) normalized to vector alone. Both KIBRA and KIBRA + DN-PKM Apl I stabilized PKM Apl III compared with pNEX control and KIBRA + DN-PKM Apl II. One-way ANOVA $\left(F_{(3,122)}=6.3, p<0.001\right)$ with Tukey's post hoc test: ${ }^{* *} p<$ 0.01 , compared with control; * $p<0.05$ (over bar), compared with KIBRA + DN-PKM Apl II. pNEX, $n=29$ neurons; KIBRA, $n=27$ neurons; KIBRA + DN-PKM AplI, $n=31$ neurons; KIBRA + DN-PKM Apl II, $n=39$ neurons; neurons from four independent experiments. Error bars indicate SEM.

KIBRA's stabilization of PKM Apl III, supporting the hypothesis that the reversal of associative LTF when DN-PKM Apl II is overexpressed in the motor neuron (Hu et al., 2017b) is due to competition with PKM Apl III for KIBRA-mediated stabilization and not the DN's influence on endogenous PKM Apl II.

\section{A KIBRA splice variant stabilizes PKM Apl I, but not PKM Apl III}

The mutant KIBRA (KIBRA-AAA) is able to stabilize PKM Apl I when both are overexpressed in cultured sensory neurons, a stabilization that is not seen with the unmutated KIBRA (Hu et al., 2017b). We hypothesized that this mutation led to a conformational change within the KIBRA protein that opened up a binding site for PKM Apl I. Examination of homology among KIBRAs over evolution reveals three homology regions: the WW domains, the $\mathrm{C} 2$ domain, and a third region beginning before the sequence identified as binding to PKMs and spanning $\sim 200$ amino acids that has not previously been defined as a domain and is not similar to other defined domains. We are naming this region the KIBRA specific domain (KSD) (Fig. 7A). The KSD is strongly conserved in all three families of bilaterians (Fig. 7B) and shows around the same percent identity as the WW and C2 domains (Fig. 7C). The KSD can also be identified in prebilaterians, such as the sea anemone Nematostella; but compared with the C2 and WW domains, the KSD is poorly conserved in prebilaterians (Fig. 7C). Nevertheless, the amino acids required for PKM $\zeta$ binding are still present in the prebilaterians (Fig. $7 B$ ). Interestingly, neither KIBRA nor the KSD is present in earlier prebilaterians 
A

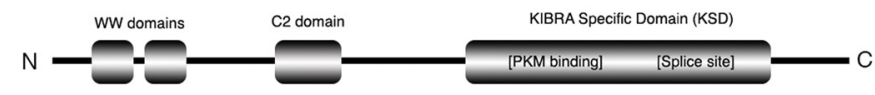

\section{B}

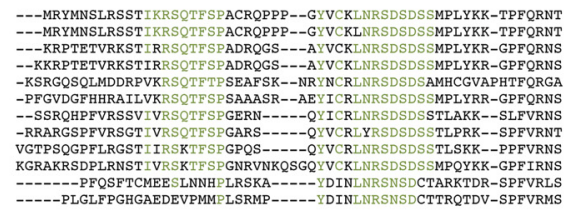

PKM
binding

VSRRSLRWKRADGSVGFMPLTSHTPF------RTSLDLEIDLOASHTKLSHLTDEISRI VSRRSLRWKRADGSVGFMPLTSHIPF-------RTSLDLELDLQASHTKLSHLTDEISRLK TVROSLRWKRLPGAAGSS--LRKMPI-------RTSVDLEIDLQASQRRLNHLRDDVYRL AERRSLRFHSKAPKSVTKLHH-------THIPRTSLDLELDLQAQHSKLYFLNDQIAKLQ
VERRSLRWRASSLAASAAAHRKSRGHPATLPPRTSVDLETDLLAONKRLOELHEELSKL. TERRSLRVKRTVCOS---VLRRTTOEC---PVRTSLDLELDLOASLTROSRLNDELOAL LERRTLRYKQSCRS--------SLAEL---MARTSLDLELDLQASRTRQRQLNEELCALI LERRSURMKRPSPPPQPSSVKSLRSER---LIRTSLDLELDLQATRTWHSQLTQEISVV MERSSMRRKKRPVSWLGVOOYOKL OMMOSLPFDMFRYSDLENDQ-DIMPROTSGDIVRL AERTSMRRKKRPVSWQGLQQYQQLSPLFGD-----GNSKSATVA-ELDVHQEASRAKQL

Splice

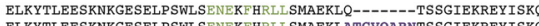
ELKYTLEESKNKGESELPSWLSENEKFHRLISMAAEKLATCVQARNTSSGIEKREYISKO ELKKGMEEAKARGANELPSWLTDDENEQRLISEADKL---------AHKSENDPDNSKQ LKKKGME EAKA CANG ELKRRMEDARYLGNODLPAWLSEDDRLQALIEHAEKRKE----------EKTVERRK DLRQKLEELKAQGETDLPPGVLEDER QRIIKQAERQAE-----------QSKEEQKQG ILRQRLEDAQLRGQTDLPPWLRDERLRGLLREAERQTR-----------OTKLDYRHE LTKEOLEQAKSHGEKELPOWLREDER RRLLLRMLEKRQ-----------MDRAEHKG RLKQWIEKALADGSTDVPKWLEDSEEYRSLVREIEGRQMRLPR-------VARRNHKAA
QLKKILEEAMGGLTEVPKLLEENDEFQSLIRDVEGRSWWILH-------GHRGRIPFS

DKRAEHLMKKVTKEVQRMRQ---GTQQSRMFT RREKMAFFTSANMDVPVIPSEDKRAEHLMKKVTKEVQRMRQ---GTQQSRMFT RREKMAFFT SANMDVPVIPSENKRAEQIMKKVTRDVEKIQK---NYFPNAFSCTHSCLWLIGCAPVDA------NKRAEQIIKKVIRDVEKIOK---NYFPNAFSCTHSCLWLIGCAPVDA------

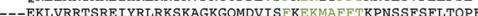
-LNAEKLMRQVSKDVCRLRE-QSOKVPRQVQSEREKIAYFTRAKISIPSLPAD-QAAEKMLKKASKETYQLRG-QSHKEPIQVQTFREKIAFFTRPRINIPPLPAD- LQTDKMMRAAAKDVHRLRG-OSCKEPEEVQSPREKMAFFTRPRMNIPALSADRRNHKVLRERLNPPPSKPQKSSATPNGSEHWV-

\section{C \\ Conservation of domains in ApKIBRA to other species percent identity/ percent similarity}

\begin{tabular}{|l|l|l|l|}
\hline Species & WW domains & C2 domain & KSD \\
\hline Octopus & $67 \% / 84 \%$ & $54 \% / 74 \%$ & $60 \% / 76 \%$ \\
\hline Drosophila & $45 \% / 66 \%$ & $46 \% / 61 \%$ & $39 \% / 58 \%$ \\
\hline Human & $47 \% / 69 \%$ & $41 \% / 56 \%$ & $43 \% / 54 \%$ \\
\hline Nematostella & $38 \% / 58 \%$ & $42 \% / 56 \%$ & $15 \% / 35 \%$ \\
\hline
\end{tabular}

D
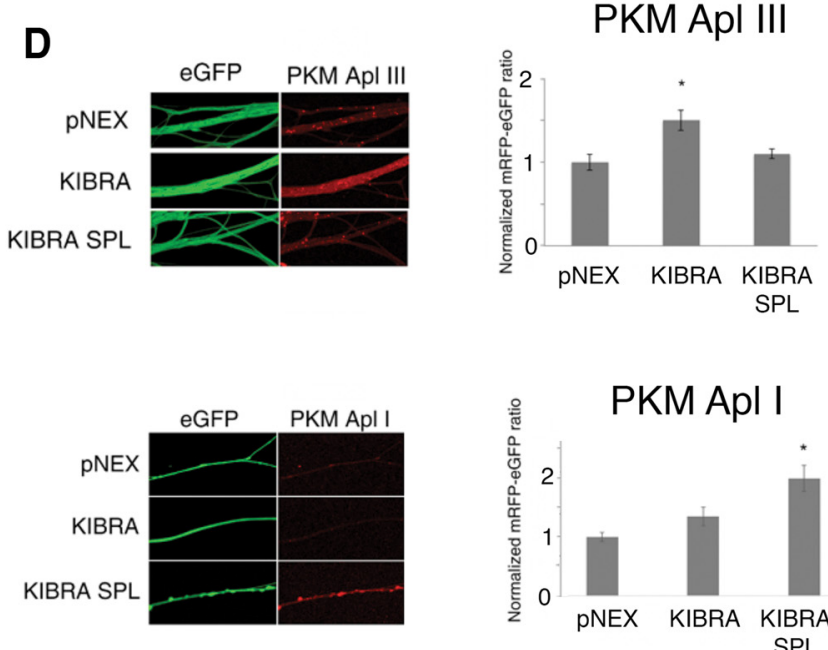

Figure 7. KIBRA Splice (SPL) stabilizes PKM ApI I, but not PKM ApI III. A, Schematic of KIBRA sequence highlighting the three conserved domains and the placement of the putative PKM binding site and the splice. $\boldsymbol{B}$, Alignment of the KSD domain from representative bilaterian and prebilaterian animals. Purple represents alternative mRNA sequences identified by bioinformatics (accession numbers below). Red represents the three amino acids required for PKM binding. Green represents five regions of increased homology. In vertebrates, the KIBRA gene has two additional paralogs, termed WWC2 and WWC3 (KIBRA's alternative name is WWC1), and all three are included in the alignment. The primary and alternate sequences with splices were derived from the following sources: Aplysia (Ap) XP_012936697.1 1288:1503; Aplysia splice gene tools: octopus (Oc) XP_014775126.1 1038:1245; octopus splice K0F84653.1 723:904; Drosophila (Dr) NP_001034055.1 966:1156; Daphnia (Da) EFX86200.1876:1090; Amphioxus (Am) XP_019625123.1995:1209; human (Ho) WWC2XP_024309993.1,913:1116 human WWC3 NP_056506.2891:1090; human KIBRA XP_016864767.1 821:1028I human Kibra lacking splice PPQPS XP_005265907.1, human KIBRA lacking Q XP_011532791.1; Nematostella (Anemone, Ne); XP_001629271.1 911:1121, Acropora (Coral; Ac) XP_015763151.1 950:1136. C, Table of homology between the Aplysia WW, C2 and KSD domains and representative species from mollusks (octopus), two other bilaterians (human, Drosophila) and a prebilaterian (Nematostella). First number indicates percent identity, and second number indicates percent similarity from Prot blast at NCBI using Aplysia sequence as probe. $\boldsymbol{D}$, Representative images of cultured Aplysia sensory neurons expressing eGFP, mRFP-tagged PKM Apl III (top) or PKM Apl I (bottom), and KIBRA, KIBRA SPL, or empty vector (pNEX3). Left, Quantification of stabilization of PKM ApI III (top) and PKM ApI I (bottom). All results are normalized within each experiment to the average mRFP/eGFP ratio of vector alone. KIBRA stabilized PKM ApI III compared with pNEX and KIBRA SPL groups (one-way ANOVA: $F_{(2,116)}=6.8 . p<0.01$ with Tukey's post hoc test). ${ }^{*} p<0.05$, different from all other groups (pNEX, $n=36$ neurons; KIBRA, $n=50$ neurons; KIBRA SPL, $n=32$ neurons; neurons from three independent experiments). KIBRA SPL stabilized PKM Apl I compared with pNEX and KIBRA groups (one-way ANOVA: $F_{(2,68)}=6.2$, $p<0.001$ with Tukey's post hoc test). ${ }^{*} p<0.01$, compared with all groups (pNEX, $n=21$ neurons; KIBRA, $n=25$ neurons; KIBRA SPL, $n=24$ neurons; neurons from three independent experiments). Error bars indicate SEM.

(sponge, Trichoplax, choanoflagellate) that lack nervous systems (see Materials and Methods). In Aplysia, the KSD also includes an alternative splicing event that is conserved throughout molluscs (Fig. $7 B$ ). The KSD also contains two alternative inserts in human KIBRA (Fig. $7 B$, purple). To determine whether this splicing event could explain isoform specificity of KIBRA binding, we overexpressed a construct containing this splice variant (KIBRA SPL) and assessed the ability of this isoform to stabilize PKM Apl I and PKM Apl III in cultured Aplysia sensory neurons. KIBRA SPL does not stabilize PKM Apl III, but it does stabilize PKM Apl I, a pattern of stabilization identical to that of our KIBRA-AAA mutant (Fig. 7D). This suggests that production of this splice variant of KIBRA can lead to stabilization of the PKM Apl I isoform, which may be important for the maintenance of nonassociative LTF.

\section{Isoform specificity is determined by the "handle" helix of PKMs}

The isoform specificity of stabilization by KIBRA between PKM Apl I and PKM Apl III must be due to specific sequence differences between the isoforms. We examined the catalytic domains of PKCs for conserved isoform-specific differences (Fig. 8A) that were available for binding based on the known crystal structures of these domains (Fig. $8 B$ ). We chose the CT region, already known to bind to proteins with PDZ domains, such as PICK 1 (Wan et al., 2012) and a region including an $\alpha$-helix (helix 5 or 
A

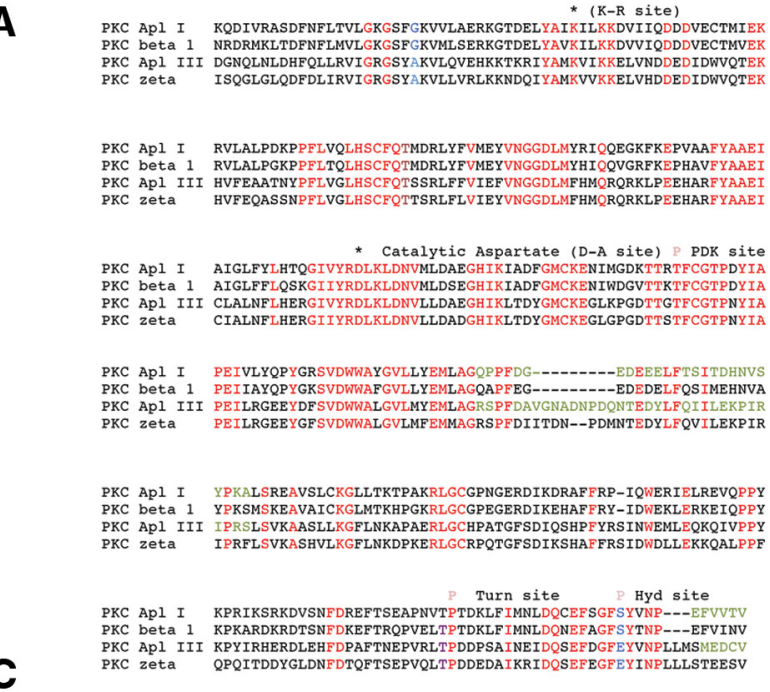

eGFP

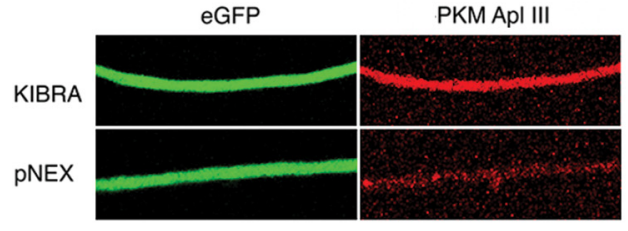

eGFP

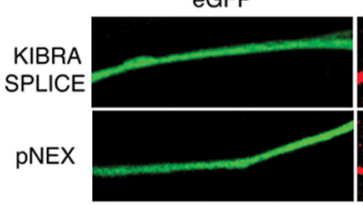

PKM ApI III

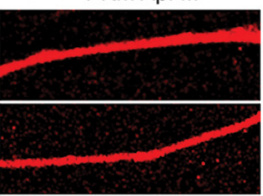

B

PKC beta II

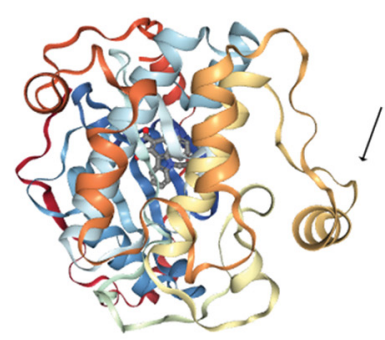

PKC iota

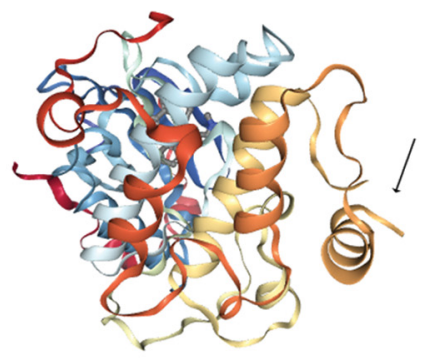

PKM ApI III CT-PKM Apl I

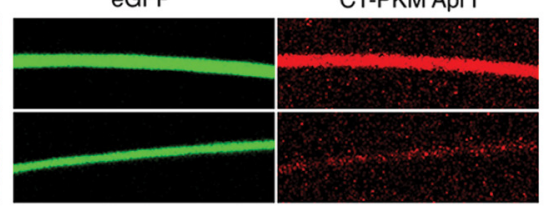

PKM ApI III

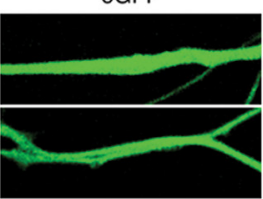

CT-PKM Apl I

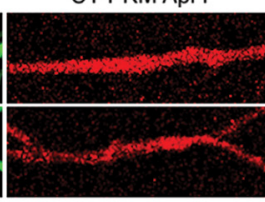

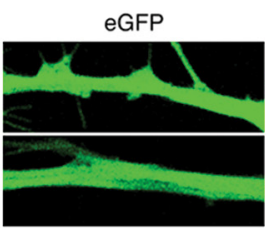

PKM ApI III handle-PKM Apl I
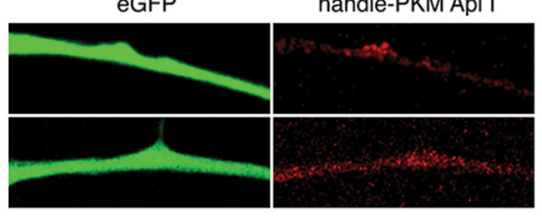

PKM ApI III handle-PKM Apl I

D

E
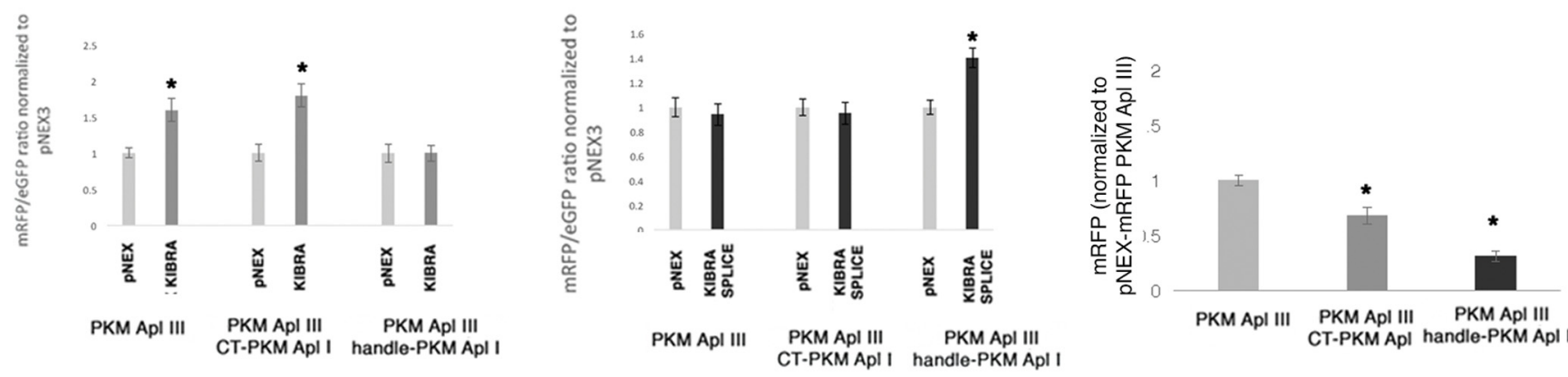

Figure 8. The "handle" domain of PKMs determines isoform-specific stabilization by KIBRA. A, Sequences of catalytic domains from classical and atypical PKCs from Aplysia and human are shown to illustrate regions of homology. Red amino acids are conserved between isoforms. Blue amino acids represent known important differences, a glycine in the ATP binding domain that partially explains the difference in ATP-based inhibitors between atypical and classical PKCs and the carboxy-terminal hydrophobic (Hyd) phosphorylation site in classical PKCs that is a glutamic acid in atypical PKCS. The sites mutated to form catalytically inactive DNs are illustrated. The green residues are the amino acids removed from PKM ApI III and replaced by the green residues in PKM Apl I in the chimeras. $\boldsymbol{B}$, Rotated structures of the human atypical PKC $\iota$ isoform (Messerschmidt et al., 2005) and classical PKC $\beta \|$ isoform (Grodsky et al., 2006) to illustrate the $\alpha$ helix switched in the chimera. From this orientation, the helix appears as a "handle." C, Representative images of neurites from cultured Aplysia sensory neurons. All neurons expressing eGFP. Top, KIBRA or empty vector (pNEX3). Bottom, KIBRA SPLICE or empty vector (pNEX3). Left to right, mRFP-tagged PKM Apl III, PKM ApI III-CT PKM Apl I, or PKM ApI III-handle PKM Apl I. D, The ability of KIBRA to stabilize the different PKMs was determined by a Student paired $t$ test between mRFP/eGFP ratios normalized to the $\mathrm{pNEX}$ control for each construct. Results were corrected by a Bonferroni test for multiple $t$ tests ( 6 for experiments in $\boldsymbol{D}) .{ }^{*} p<0.01$. Left, PKM Ap I III: pNEX, $n=30$ neurons; KIBRA, $n=15$ neurons; PKM ApI III-CT PKM ApI I: pNEX, $n=17$ neurons; KIBRA, $n=32$ neurons; PKM Apl III-handle PKM Ap I: pNEX, $n=14$ neurons; KIBRA, $n=33$ neurons (results are from three independent experiments with all six groups). Right, PKM ApI III: pNEX, $n=23$ neurons; KIBRA SPL, $n=19$ neurons; PKM ApI III-CT PKM ApI I: pNEX, $n=33$ neurons; KIBRA SPL, $n=23$ neurons; PKM ApI III-handle PKM ApI I: pNEX, $n=20$ neurons; KIBRA SPL $n=29$ neurons (results are from three independent experiments with all six groups). $\boldsymbol{E}$, The levels of expression of the three constructs in the absence of KIBRA were quantified. The mRFP values are normalized to levels of PKM ApI III in each experiment. One-way ANOVA $\left(F_{(2,135)}=24.5\right.$ with Tukey's post hoc test). ${ }^{*} p<0.01$, all groups different from each other (PKM Apl III, $n=53$ neurons; PKM Apl III-CT PKM Apl I, $n=51$ neurons; PKM Apl III-handle PKM Apl I, $n=34$ neurons; results are from 12 independent experiments with all three groups). 
Table 1. Presynaptic or postsynaptic overexpression of each PKM isoform produces different changes in synaptic strength ${ }^{a}$

\begin{tabular}{lllll}
\hline & PKM Apl I & PKM Apl II & PKM Apl III & KIBRA \\
\hline Presynaptic & Toxic & Toxic & No change & ND \\
Postsynaptic (L7) & Increase $^{b}$ & Increase $^{b}$ & Increase & ND \\
Postsynaptic (LFS) & ND & ND & Increase & No change \\
\hline
\end{tabular}

${ }^{a}$ Summary of data presented in Figures 1 and 2 showing the change in synaptic strength $24 \mathrm{~h}$ following injection with each of the PKM isoforms. Overexpression of PKM Apl I and PKM Apl II in LFS motor neurons was not examined. ND, Not determined.

${ }^{b}$ Toxicity was observed at time points beyond $24 \mathrm{~h}$.

helix $\mathrm{G}$ in the catalytic domain structure) that appears as a handle in the crystal structures of PKMs (Fig. 8B). We then made chimeras, exchanging these two regions (highlighted in green in Fig. $8 A$ ) from the PKM Apl III sequence for the corresponding PKM Apl I sequence, generating PKM Apl III-CT PKM Apl I and PKM Apl III-handle PKM Apl I. We then examined the ability of KIBRA and KIBRA SPL to stabilize these chimeric PKMs. PKM Apl III-CT PKM Apl I behaved like PKM Apl III in that it was stabilized by KIBRA but not by KIBRA SPL (Fig. 8C,D). In contrast, when the handle region of PKM Apl III was replaced with the handle region of PKM Apl I, stabilization was similar to PKM Apl I: the chimera was stabilized by KIBRA SPL but not by KIBRA (Fig. 8C,D). While the chimeras were expressed at lower levels than the WT PKM Apl III (Fig. 8E), the differential stabilization by the two KIBRA splice isoforms indicates that the level of expression does not explain the lack of stabilization. These results indicate that isoform specificity of PKMs is determined by the sequence in or around the handle helix.

\section{Discussion}

Sufficiency of PKMs for increasing synaptic strength

We investigated the isoform specificity and sufficiency of PKM in regulating synaptic strength by overexpressing distinct isoforms of PKM, presynaptically and postsynaptically (Table 1). Postsynaptic overexpression of PKM was sufficient to increase synaptic strength, independent of isoform identity. PKM Apl I and PKM Apl II sufficiency in the presynaptic sensory neuron could not be determined due to their toxicity after overexpression. This toxicity is also seen in the motor neuron but is delayed compared with the effects in the sensory neuron. Toxicity may be due to a role of PKMs in inducing apoptosis as cleavage of the novel PKC $\delta$ by caspases to form a PKM is proapoptotic in many systems (Reyland, 2007). PKM Apl III overexpression is not toxic in either the sensory or motor neuron, despite a similar level of expression as judged by the mRFP level. This allows for the conclusion that PKM Apl III is sufficient in the postsynaptic cell, but not the presynaptic cell, and this result was replicated with two different motor neurons serving as the postsynaptic cell (Table 1). Despite it not being sufficient to increase synaptic strength in the presynaptic sensory neuron, there is evidence that PKM Apl III in the sensory neuron is important for nonassociative LTF in the sensory neuron. DN-PKM Apl III erases nonassociative LTF when expressed in the sensory neuron (Hu et al., 2017a), and presynaptic overexpression of a distinct DN form of PKC Apl III blocks nonassociative LTF 48 and $72 \mathrm{~h}$ after induction (Fiumara et al., 2015). Presynaptic overexpression of PKC Apl III, which leads to production of PKM Apl III (Bougie et al., 2009), was not sufficient to increase synaptic strength, similar to the present study but did lead to stronger nonassociative LTF (Fiumara et al., 2015). In conclusion, PKM Apl III in the sensory neuron is not sufficient to increase synaptic strength but is required for mediating nonassociative LTF.
Table 2. KIBRA-mediated stabilization of PKMs is isoform-specific and independent of catalytic activity ${ }^{a}$

\begin{tabular}{|c|c|c|c|c|c|c|c|c|}
\hline & \multicolumn{2}{|c|}{ PKM Apl I } & \multicolumn{2}{|c|}{ PKM Apl II } & \multicolumn{2}{|c|}{ PKM Apl III } & \multirow{2}{*}{$\begin{array}{l}\text { PKM Apl III } \\
\text { CT-PKM Apl I }\end{array}$} & \multirow{2}{*}{$\begin{array}{l}\text { PKM Apl III } \\
\text { handle-PKM ApI I }\end{array}$} \\
\hline & WT & DN & WT & DN & WT & DN & & \\
\hline KIBRA & $x^{b}$ & $X$ & $S^{b}$ & $S$ & $S^{b}$ & $S$ & $S$ & $x$ \\
\hline KIBRA-AAA & $S^{b}$ & $S$ & $S^{b}$ & $x$ & $x^{b}$ & $x$ & ND & ND \\
\hline KIBRA-Spl & $S$ & ND & ND & ND & $x$ & ND & $x$ & $S$ \\
\hline
\end{tabular}

aStabilization of WT PKM isoforms and DN-PKM isoforms by each of our KIBRA constructs. S, Stabilization; $X$, no stabilization; ND, not determined.

${ }^{b}$ Hu et al. (2017b).

\section{Requirement for PKMs in both presynaptic and postsynaptic} cell for associative LTF

While PKM Apl III postsynaptic overexpression is sufficient to increase synaptic strength, this is not sufficient to induce other forms of plasticity that accompany persistent associative LTF. Persistent associative LTF, which is induced by presynaptic activity in combination with 5-HT application in Aplysia and is a cellular analog of classical-conditioning (Buonomano and Byrne, 1990; Hu and Schacher, 2015), is characterized by a persistent increase in synaptic strength in conjunction with an attenuation of HSD kinetics (Hu and Schacher, 2015); we have shown that these two forms of plasticity are produced by presynaptic overexpression of PKC Apl I in combination with postsynaptic PKM Apl III overexpression. Interestingly, PKC Apl I is also important for burst-dependent protection from HSD (Wan et al., 2012), although the relationship between this form of plasticity and the attenuation of HSD that accompanies persistent associative LTF is not clear. Since presynaptic overexpression of DN-PKMs and DN KIBRA-AAA is sufficient to reverse different types of LTF in an isoform-specific manner (Hu et al., 2017a), isoform specificity of PKMs extends to both the presynaptic and postsynaptic side of a synapse.

\section{Failure of KIBRA overexpression to prolong m-ITF}

While the ability of overexpressed PKM to increase synaptic strength suggests that simple stabilization of the PKM produced by m-ITF (Villareal et al., 2009; Bougie et al., 2012) by KIBRA should have led to increased synaptic strength, it is likely that the levels of PKM produced by stimulation are much lower than that produced through overexpression. Simply because large amounts of PKM are sufficient to increase synaptic strength does not necessarily mean that the amounts of PKM produced endogenously are sufficient to increase synaptic strength, even when stabilized by KIBRA. Endogenous levels of PKM probably require interactions with other postsynaptic processes generated by LTF that are not induced by m-ITF to increase synaptic strength during LTF.

\section{KIBRA determines isoform specificity of PKMs}

A weakness of DN constructs is their possible lack of specificity, but this also allows investigators to probe functional aspects of the proteins examined. Overexpression of DN-PKM Apl II in the postsynaptic neuron is sufficient to reverse associative LTF (Hu et al., 2017a), suggesting that PKM Apl II may be important in the maintenance of this type of LTF. KIBRA-AAA stabilizes PKM Apl II (Hu et al., 2017b). One might assume, therefore, that overexpression of KIBRA-AAA in the postsynaptic neuron would not have an effect on associative LTF. However, this is not the case. KIBRA-AAA overexpression is sufficient to reverse associative LTF, suggesting that the ability of DN-PKM Apl II overexpression to reverse associative LTF is the result of nonspecificity of this construct. Here we explain this lack of specificity by demon- 
strating that DN-PKM Apl II interferes with KIBRA-mediated stabilization of PKM Apl III. While our results are consistent with DN-PKMs erasing LTF through competition for KIBRA binding, these experiments do not rule out that other interactions could also be important for the actions of the DN-PKMs.

Our results support a model where distinct splice isoforms of KIBRA stabilize distinct PKMs (Table 2) and thus predict that synapses expressing persistent nonassociative LTF would be enriched with splice forms of KIBRA-stabilizing PKM Apl I, whereas synapses supporting persistent associative LTF would be enriched with splice forms of KIBRA-stabilizing PKM Apl III. This model also predicts that the alternatively spliced KSD also interacts with other distinct markers of these synapses (Sossin, 2018) to specifically localize the KIBRA splice isoforms to distinct synapses. Alternatively, since distinct calpains are required for induction of associative and nonassociative LTF (Hu et al., 2017a), PKC Apl I and PKC Apl III may only be cleaved to form PKM at the appropriate synapses, and the distinct KIBRA splice isoforms could be present at all synapses. Further studies will be necessary to distinguish between these models.

\section{A new conserved region in KIBRA}

Examination of KIBRA conservation over evolution demonstrates strong conservation of a 200 amino acid region we are naming the KSD. This region includes the amino acids previously determined to be required for binding to PKM $\zeta$ (Vogt-Eisele et al., 2014), and required for the stabilization of PKM Apl III; however, these residues are not required for stabilization of PKM Apl I (Hu et al., 2017b). Along with its role in stabilizing PKMs and regulating AMPA receptor trafficking, KIBRA is also part of the Hippo signaling pathway important for the regulation of cell proliferation in development and in cancer (Genevet et al., 2010; Yu et al., 2010). While KIBRA's role in this pathway is mainly to act as a scaffold to localize members of this pathway through its WW domains (Baumgartner et al., 2010; Xiao et al., 2011; Zhang et al., 2014), several interactions have been localized to the KSD, including binding to Merlin, and dimerization (Baumgartner et al., 2010; Wennmann et al., 2014). These interactions may require the KSD.

\section{How does KIBRA stabilize PKMs?}

While the simplest model would be to assume that KIBRAmediated stabilization is simply due to direct binding, this may not be the case. KIBRA stabilizes the large tumor suppressor kinase (Lats) as part of its role in the Hippo pathway, but this stabilization can be dissociated from Lats2 binding to KIBRA as binding requires the WW domains, but stabilization does not (Xiao et al., 2011). KIBRA is a scaffold protein with many protein-protein interactions (Zhang et al., 2014), and KIBRA may also be involved in localization of PKMs. There is evidence showing an association between KIBRA and AMPA receptors (Makuch et al., 2011), as well as a general enrichment in the postsynaptic density (Johannsen et al., 2008), suggesting the possibility that KIBRA may help localize PKMs to their synaptic targets in addition to their stabilizing effect.

We have identified features of PKM that are necessary for KIBRA-mediated stabilization. Neither catalytic activity nor priming phosphorylation is required since KIBRA stabilized a DN-PKM Apl III made through a K-R mutation that prevents priming phosphorylation (Cameron et al., 2009; Bougie et al., 2012). Switching of a region, including one $\alpha$-helix, that in crystal structures resembles a handle, was sufficient to switch the specificity of stabilization by splice forms of KIBRA, suggesting that this is the key region of PKMs that determines isoform specificity (Table 2). Interestingly, ICAP and $\zeta$-stat, isoform-specific PKC inhibitors, are targeted to this region (Pillai et al., 2011; Ratnayake et al., 2018). This region also may play a role in determining isoform-specific substrate specificity of PKCs (Soriano et al., 2016).

In conclusion, PKMs have isoform- and synapse-specific roles in the maintenance of LTF. This isoform specificity may be achieved through selective stabilization by KIBRA. The isoform specificity of KIBRA-mediated stabilization of PKMs depends on the handle helix and is independent of catalytic activity of the kinase (Table 2). These results contribute to our understanding of the mechanisms underlying memory maintenance as well as the synaptic differences among different types of synaptic plasticity that underlie distinct types of memory.

\section{References}

Armitage BA, Siegelbaum SA (1998) Presynaptic induction and expression of homosynaptic depression at Aplysia sensorimotor neuron synapses. J Neurosci 18:8770-8779.

Baumgartner R, Poernbacher I, Buser N, Hafen E, Stocker H (2010) The WW domain protein Kibra acts upstream of Hippo in Drosophila. Dev Cell 18:309-316.

Bougie JK, Lim T, Farah CA, Manjunath V, Nagakura I, Ferraro GB, Sossin WS (2009) The atypical protein kinase $\mathrm{C}$ in Aplysia can form a protein kinase M by cleavage. J Neurochem 109:1129-1143.

Bougie JK, Cai D, Hastings M, Farah CA, Chen S, Fan X, McCamphill PK, Glanzman DL, Sossin WS (2012) Serotonin-induced cleavage of the atypical protein kinase C apl III in Aplysia. J Neurosci 32:14630-14640.

Buonomano DV, Byrne JH (1990) Long-term synaptic changes produced by a cellular analog of classical conditioning in Aplysia. Science 249:420423.

Cai D, Pearce K, Chen S, Glanzman DL (2011) Protein kinase M maintains long-term sensitization and long-term facilitation in Aplysia. J Neurosci 31:6421-6431.

Cameron AJ, Escribano C, Saurin AT, Kostelecky B, Parker PJ (2009) PKC maturation is promoted by nucleotide pocket occupation independently of intrinsic kinase activity. Nat Struct Mol Biol 16:624-630.

Chen S, Cai D, Pearce K, Sun PY, Roberts AC, Glanzman DL (2014) Reinstatement of long-term memory following erasure of its behavioral and synaptic expression in Aplysia. Elife 3:e03896.

Dunn TW, Sossin WS (2013) Inhibition of the Aplysia sensory neuron calcium current with dopamine and serotonin. J Neurophysiol 110:20712081.

Farah CA, Hastings MH, Dunn TW, Gong K, Baker-Andresen D, Sossin WS (2017) A PKM generated by calpain cleavage of a classical PKC is required for activity-dependent intermediate-term facilitation in the presynaptic sensory neuron of Aplysia. Learn Mem 24:1-13.

Fiumara F, Rajasethupathy P, Antonov I, Kosmidis S, Sossin WS, Kandel ER (2015) MicroRNA-22 gates long-term heterosynaptic plasticity in Aplysia through presynaptic regulation of CPEB and downstream targets. Cell Rep 11:1866-1875.

Genevet A, Wehr MC, Brain R, Thompson BJ, Tapon N (2010) Kibra is a regulator of the Salvador/Warts/Hippo signaling network. Dev Cell 18: 300-308.

Greenberg SM, Castellucci VF, Bayley H, Schwartz JH (1987) A molecular mechanism for long-term sensitization in Aplysia. Nature 329:62-65.

Grodsky N, Li Y, Bouzida D, Love R, Jensen J, Nodes B, Nonomiya J, Grant S (2006) Structure of the catalytic domain of human protein kinase $\mathrm{C}$ beta II complexed with a bisindolylmaleimide inhibitor. Biochemistry 45: $13970-13981$.

Hastings MH, Qiu A, Zha C, Farah CA, Mahdid Y, Ferguson L, Sossin WS (2018) The zinc fingers of the small optic lobes calpain bind polyubiquitin. J Neurochem 146:429-445.

Heitz FD, Farinelli M, Mohanna S, Kahn M, Duning K, Frey MC, Pavenstädt H, Mansuy IM (2016) The memory gene KIBRA is a bidirectional regulator of synaptic and structural plasticity in the adult brain. Neurobiol Learn Mem 135:100-114.

Hu J, Schacher S (2015) Persistent associative plasticity at an identified syn- 
apse underlying classical conditioning becomes labile with short-term homosynaptic activation. J Neurosci 35:16159-16170.

$\mathrm{Hu}$ JY, Schacher S (2014) Persistent long-term facilitation at an identified synapse becomes labile with activation of short-term heterosynaptic plasticity. J Neurosci 34:4776-4785.

Hu J, Adler K, Farah CA, Hastings MH, Sossin WS, Schacher S (2017a) Cell-specific PKM isoforms contribute to the maintenance of different forms of persistent long-term synaptic plasticity. J Neurosci 37:27462763.

Hu J, Ferguson L, Adler K, Farah CA, Hastings MH, Sossin WS, Schacher S (2017b) Selective erasure of distinct forms of long-term synaptic plasticity underlying different forms of memory in the same postsynaptic neuron. Curr Biol 27:1888-1899.e4.

Johannsen S, Duning K, Pavenstädt H, Kremerskothen J, Boeckers TM (2008) Temporal-spatial expression and novel biochemical properties of the memory-related protein KIBRA. Neuroscience 155:1165-1173.

Kaang BK (1996) Parameters influencing ectopic gene expression in Aplysia neurons. Neurosci Lett 221:29-32.

Lisman J (1994) The CaM kinase II hypothesis for the storage of synaptic memory. Trends Neurosci 17:406-412.

Livak KJ, Schmittgen TD (2001) Analysis of relative gene expression data using real-time quantitative PCR and the 2(-delta delta $\mathrm{C}(\mathrm{T})$ ) method. Methods 25:402-408.

Makuch L, Volk L, Anggono V, Johnson RC, Yu Y, Duning K, Kremerskothen J, Xia J, Takamiya K, Huganir RL (2011) Regulation of AMPA receptor function by the human memory-associated gene KIBRA. Neuron 71: 1022-1029.

McCamphill PK, Farah CA, Anadolu MN, Hoque S, Sossin WS (2015) Bidirectional regulation of eEF2 phosphorylation controls synaptic plasticity by decoding neuronal activity patterns. J Neurosci 35:4403-4417.

Messerschmidt A, Macieira S, Velarde M, Bädeker M, Benda C, Jestel A, Brandstetter H, Neuefeind T, Blaesse M (2005) Crystal structure of the catalytic domain of human atypical protein kinase $\mathrm{C}$-iota reveals interaction mode of phosphorylation site in turn motif. J Mol Biol 352:918 -931.

Milnik A, Heck A, Vogler C, Heinze HJ, de Quervain DJ, Papassotiropoulos A (2012) Association of KIBRA with episodic and working memory: a meta-analysis. Am J Med Genet B Neuropsychiatr Genet 159B:958-969.

Papassotiropoulos A, Stephan DA, Huentelman MJ, Hoerndli FJ, Craig DW, Pearson JV, Huynh KD, Brunner F, Corneveaux J, Osborne D, Wollmer MA, Aerni A, Coluccia D, Hänggi J, Mondadori CR, Buchmann A, Reiman EM, Caselli RJ, Henke K, de Quervain DJ (2006) Common Kibra alleles are associated with human memory performance. Science 314:475-478.

Pillai P, Desai S, Patel R, Sajan M, Farese R, Ostrov D, Acevedo-Duncan M (2011) A novel PKC- $\iota$ inhibitor abrogates cell proliferation and induces apoptosis in neuroblastoma. Int J Biochem Cell Biol 43:784-794.

Ratnayake WS, Apostolatos CA, Apostolatos AH, Schutte RJ, Huynh MA, Ostrov DA, Acevedo-Duncan M (2018) Oncogenic PKC- $\iota$ activates vimentin during epithelial-mesenchymal transition in melanoma: a study based on PKC- $\iota$ and PKC- $\zeta$ specific inhibitors. Cell Adh Migr 12:447463.

Reyland ME (2007) Protein kinase $c \delta$ and apoptosis. Biochem Soc Trans 35:1001-1004.

Sacktor TC (2011) How does PKM $\zeta$ maintain long-term memory? Nat Rev Neurosci 12:9-15.

Shema R, Haramati S, Ron S, Hazvi S, Chen A, Sacktor TC, Dudai Y (2011)
Enhancement of consolidated long-term memory by overexpression of protein kinase $\mathrm{M} \zeta$ in the neocortex. Science 331:1207-1210.

Soriano EV, Ivanova ME, Fletcher G, Riou P, Knowles PP, Barnouin K, Purkiss A, Kostelecky B, Saiu P, Linch M, Elbediwy A, Kjaer S, O’Reilly N, Snijders AP, Parker PJ, Thompson BJ, McDonald NQ (2016) aPKC inhibition by Par3 CR3 flanking regions controls substrate access and underpins apical-junctional polarization. Dev Cell 38:384-398.

Sossin WS (2007) Isoform specificity of protein kinase Cs in synaptic plasticity. Learn Mem 14:236-246.

Sossin WS (2018) Memory synapses are defined by distinct molecular complexes: a proposal. Front Synaptic Neurosci 10:5.

Sutton MA, Ide J, Masters SE, Carew TJ (2002) Interaction between amount and pattern of training in the induction of intermediate- and long-term memory for sensitization in Aplysia. Learn Mem 9:29-40.

Tsokas P, Hsieh C, Yao Y, Lesburguères E, Wallace EJ, Tcherepanov A, Jothianandan D, Hartley BR, Pan L, Rivard B, Farese RV, Sajan MP, Bergold PJ, Hernández AI, Cottrell JE, Shouval HZ, Fenton AA, Sacktor TC (2016) Compensation for PKM $\zeta$ in long-term potentiation and spatial long-term memory in mutant mice. Elife 5:e14846.

Villareal G, Li Q, Cai D, Fink AE, Lim T, Bougie JK, Sossin WS, Glanzman DL (2009) Role of protein kinase $C$ in the induction and maintenance of serotonin-dependent enhancement of the glutamate response in isolated siphon motor neurons of Aplysia californica. J Neurosci 29:5100-5107.

Vogt-Eisele A, Krüger C, Duning K, Weber D, Spoelgen R, Pitzer C, Plaas C, Eisenhardt G, Meyer A, Vogt G, Krieger M, Handwerker E, Wennmann DO, Weide T, Skryabin BV, Klugmann M, Pavenstädt H, Huentelmann MJ, Kremerskothen J, Schneider A (2014) KIBRA (KIdney/BRAin protein) regulates learning and memory and stabilizes protein kinase $\mathrm{M} \zeta$. J Neurochem 128:686-700.

Wan Q, Jiang XY, Negroiu AM, Lu SG, McKay KS, Abrams TW (2012) Protein kinase $\mathrm{C}$ acts as a molecular detector of firing patterns to mediate sensory gating in Aplysia. Nat Neurosci 15:1144-1152.

Wennmann DO, Schmitz J, Wehr MC, Krahn MP, Koschmal N, Gromnitza S, Schulze U, Weide T, Chekuri A, Skryabin BV, Gerke V, Pavenstädt H, Duning K, Kremerskothen J (2014) Evolutionary and molecular facts link the WWC protein family to Hippo signaling. Mol Biol Evol 31:1710-1723.

Xiao L, Chen Y, Ji M, Dong J (2011) KIBRA regulates Hippo signaling activity via interactions with large tumor suppressor kinases. J Biol Chem 286:7788-7796

Yao Y, Kelly MT, Sajikumar S, Serrano P, Tian D, Bergold PJ, Frey JU, Sacktor TC (2008) PKM $\zeta$ maintains late long-term potentiation by N-ethylmaleimide-sensitive factor/GluR2-dependent trafficking of postsynaptic AMPA receptors. J Neurosci 28:7820-7827.

Yoshihama Y, Hirai T, Ohtsuka T, Chida K (2009) KIBRA co-localizes with protein kinase $\mathrm{M} \zeta(\mathrm{PKM} \zeta)$ in the mouse hippocampus. Biosci Biotechnol Biochem 73:147-151.

Yu J, Zheng Y, Dong J, Klusza S, Deng WM, Pan D (2010) Kibra functions as a tumor suppressor protein that regulates Hippo signaling in conjunction with Merlin and expanded. Dev Cell 18:288-299.

Zhang L, Yang S, Wennmann DO, Chen Y, Kremerskothen J, Dong J (2014) KIBRA: in the brain and beyond. Cell Signal 26:1392-1399.

Zhao Y, Leal K, Abi-Farah C, Martin KC, Sossin WS, Klein M (2006) Isoform specificity of PKC translocation in living Aplysia sensory neurons and a role for $\mathrm{Ca}^{2+}$-dependent PKC APL I in the induction of intermediate-term facilitation. J Neurosci 26:8847-8856. 